\title{
Spaces of surface group representations
}

\author{
Kathryn Mann
}

\begin{abstract}
Let $\Gamma_{g}$ denote the fundamental group of a closed surface of genus $g \geq 2$. We show that every geometric representation of $\Gamma_{g}$ into the group of orientation-preserving homeomorphisms of the circle is rigid, meaning that its deformations form a single semi-conjugacy class.

As a consequence, we give a new lower bound on the number of topological components of the space of representations of $\Gamma_{g}$ into Homeo $+\left(S^{1}\right)$. Precisely, for each nontrivial divisor $k$ of $2 g-2$, there are at least $k^{2 g}+1$ components containing representations with Euler number $\frac{2 g-2}{k}$. Our methods apply to representations of surface groups into finite covers of $\operatorname{PSL}(2, \mathbb{R})$ and into $\operatorname{Diff}_{+}\left(S^{1}\right)$ as well, in which case we recover theorems of W. Goldman and J. Bowden.

The key technique is an investigation of stability phenomena for rotation numbers of products of circle homeomorphisms using techniques of Calegari-Walker. This is a new approach to studying deformation classes of group actions on the circle, and may be of independent interest.
\end{abstract}

\section{Introduction}

Let $\Gamma_{g}$ denote the fundamental group of the closed genus $g$ surface $\Sigma_{g}$, and let $G$ be a group of orientation-preserving homeomorphisms of the circle. The representation space $\operatorname{Hom}\left(\Gamma_{g}, G\right)$ is a basic object in geometry and topology: it parametrizes flat circle bundles over $\Sigma_{g}$ with structure group $G$, as well as actions of $\Gamma_{g}$ on the circle with degree of regularity specified by $G$ (e.g. $G=\operatorname{Diff}^{r}\left(S^{1}\right)$ for $C^{r}$-regularity). A fundamental question is to identify and characterize the deformation classes of $\Gamma_{g}$-actions on $S^{1}$; equivalently, the deformation classes of flat $S^{1}$ bundles with a given structure group, or the connected components of $\operatorname{Hom}\left(\Gamma_{g}, G\right)$.

When $G \subset \operatorname{Homeo}_{+}\left(S^{1}\right)$ is a transitive Lie group, the space $\operatorname{Hom}\left(\Gamma_{g}, G\right)$ is quite well understood, and the Milnor-Wood inequality together with work of W. Goldman gives a complete classification of its connected components using the Euler number. Far less is known when $G$ is not a Lie group, and the problem of classifying components of $\operatorname{Hom}\left(\Gamma_{g}, G\right)$ for $G=\operatorname{Homeo}_{+}\left(S^{1}\right)$ is essentially completely open. The purpose of this paper is to develop a new approach to distinguish components of representation spaces, applicable to the $G=$ Homeo+ $_{+}\left(S^{1}\right)$ case.

Our approach also gives techniques to study rigidity of representations. We are particularly interested in geometric representations, in the following sense.

Definition 1.1. Let $M$ and $X$ be manifolds. A representation $\rho: \pi_{1}(M) \rightarrow \operatorname{Homeo}_{+}(X)$ is geometric if it is faithful and has discrete image contained in a finite-dimensional Lie group $G \subset \mathrm{Homeo}_{+}(X)$ acting transitively on $X$.

For example, any complete $(G, X)$ structure on a manifold $M$ (in the sense of Thurston) defines a geometric representation $\rho: \pi_{1}(M) \rightarrow G \subset$ Homeo $_{+}(X)$.

We are interested in the case $M=\Sigma_{g}$ and $X=S^{1}$. We will show that all geometric representations $\Gamma_{g}=\pi_{1}\left(\Sigma_{g}\right) \rightarrow \operatorname{Homeo}\left(S^{1}\right)$ are rigid, in the sense that they admit only trivial deformations.

Theorem 1.2 (Rigidity of geometric representations). Let $g \geq 2$, and let $\rho$ be a geometric representation in $\operatorname{Hom}\left(\Gamma_{g}, \operatorname{Homeo}_{+}\left(S^{1}\right)\right)$. Then the connected component of $\rho$ in $\operatorname{Hom}\left(\Gamma_{g}, \mathrm{Homeo}_{+}\left(S^{1}\right)\right)$ consists of a single semi-conjugacy class. 
From here on, $g \geq 2$ will be a standing assumption.

Rigidity of geometric representations gives the following new lower bound on the number of components of $\operatorname{Hom}\left(\Gamma_{g}, \mathrm{Homeo}_{+}\left(S^{1}\right)\right)$. In particular, we can show - in contrast to the classical $G=\operatorname{PSL}(2, \mathbb{R})$ case - that components of $\operatorname{Hom}\left(\Gamma_{g}, \operatorname{Homeo}_{+}\left(S^{1}\right)\right)$ are not distinguished by the Euler number.

Theorem 1.3 (Lower bound). Let $k$ be a nontrivial divisor of $2 g-2$. There are at least $k^{2 g}+1$ components of $\operatorname{Hom}\left(\Gamma_{g}\right.$, Homeo+ $\left.\left(S^{1}\right)\right)$ consisting of representations with Euler number $\frac{2 g-2}{k}$.

The primary tool in the proofs of Theorems 1.3 and 1.2, suggested to the author by D. Calegari, is the study of rotation numbers of elements in the image of a representation $\rho$. (Rotation number is a classical invariant; we will recall the definition in Section 2.) For each $\gamma \in \Gamma_{g}$, define $\operatorname{rot}_{\gamma}: \operatorname{Hom}\left(\Gamma_{g}\right.$, Homeo $\left._{+}\left(S^{1}\right)\right) \rightarrow \mathbb{R} / \mathbb{Z}$ by $\operatorname{rot}_{\gamma}(\rho)=\operatorname{rot}(\rho(\gamma))$, where $\operatorname{rot}(\cdot)$ denotes rotation number. We prove the following strong form of rigidity.

Theorem 1.4 (Rotation number rigidity). Let $\gamma \in \Gamma_{g}$ and let $X \subset \operatorname{Hom}\left(\Gamma_{g}, \operatorname{Homeo}_{+}\left(S^{1}\right)\right)$ be a connected component containing a geometric representation. Then $\operatorname{rot}_{\gamma}$ is constant on $X$.

\subsection{Known results on representation spaces $\operatorname{Hom}\left(\Gamma_{g}, G\right)$}

To give context to our work, we briefly summarize known results describing the representation spaces $\operatorname{Hom}\left(\Gamma_{g}, G\right)$ for various $G \subset \operatorname{Homeo}_{+}\left(S^{1}\right)$.

\section{Lie group case}

When $G \subset \operatorname{Homeo}\left(S^{1}\right)$ is a Lie group, $\operatorname{Hom}\left(\Gamma_{g}, G\right)$ has the structure of an affine variety and hence has only finitely many components. The interesting case is when $G$ acts transitively on $S^{1}$, in which case $G$ must either be $S^{1}$ or the $k$-fold cyclic cover $\operatorname{PSL}^{(\mathrm{k})}$ of $\operatorname{PSL}(2, \mathbb{R})$ for some $k \geq 1$. It is easy to see that $\operatorname{Hom}\left(\Gamma_{g}, S^{1}\right)$ is connected, and the components of $\operatorname{Hom}\left(\Gamma_{g}, \mathrm{PSL}^{(\mathrm{k})}\right)$ are completely classified by the following theorem.

Theorem 1.5 (Goldman [6], using also Milnor [7]). Let $G$ be the $k$-fold cyclic cover PSL ${ }^{(\mathrm{k})}$ of $\operatorname{PSL}(2, \mathbb{R}), k \geq 1$. The connected components of $\operatorname{Hom}\left(\Gamma_{g}, G\right)$ are completely classified by

a) The Euler number of the representation. This assumes each value between $-\left\lfloor\frac{2 g-2}{k}\right\rfloor$ and $\left\lfloor\frac{2 g-2}{k}\right\rfloor$ and is constant on connected components. Each value of the Euler number is assumed on a single connected component, with the sole exception of b) below.

b) In the case where $2 g-2=n k$ for some integer $n$, there are $k^{2 g}$ connected components of representations with Euler number $n$, and $k^{2 g}$ with Euler number $-n$. These components are distinguished by the rotation numbers of a standard set of generators for $\Gamma_{g}$.

In the Lie group case, geometric representations are precisely the discrete, faithful ones. It follows from Goldman's work that $\rho \in \operatorname{Hom}\left(\Gamma_{g}, \mathrm{PSL}^{(\mathrm{k})}\right)$ is discrete and faithful if and only if the Euler number of $\rho$ is $\frac{ \pm(2 g-2)}{k}$. See Section 2.3 below.

\section{Non-Lie groups}

When $G$ is not a Lie group (for instance, $G=$ Homeo $_{+}\left(S^{1}\right)$ or $G=\operatorname{Diff}_{+}\left(S^{1}\right)$ ), describing the space $\operatorname{Hom}\left(\Gamma_{g}, G\right)$ and classifying its components is more challenging. Though there are still only finitely many possible values for the Euler number (this is the Milnor-Wood inequality, see Section 2.2), it is not known whether $\operatorname{Hom}\left(\Gamma_{g}, \operatorname{Homeo}_{+}\left(S^{1}\right)\right)$ has finitely many or infinitely many components. On the other hand, it is possible a priori that $\operatorname{Hom}\left(\Gamma_{g}, \mathrm{Homeo}_{+}\left(S^{1}\right)\right)$ could be in a sense "more connected" than $\operatorname{Hom}\left(\Gamma_{g}, \mathrm{PSL}^{(\mathrm{k})}\right)$ - two representations into PSL ${ }^{(\mathrm{k})}$, both with Euler number $\frac{2 g-2}{k}$, but lying in different components of $\operatorname{Hom}\left(\Gamma_{g}, \mathrm{PSL}^{(\mathrm{k})}\right)$, could potentially lie in the same component of $\operatorname{Hom}\left(\Gamma_{g}, \mathrm{Homeo}_{+}\left(S^{1}\right)\right)$.

Bowden recently showed that this kind of additional connectedness does not hold for $\operatorname{Hom}\left(\Gamma_{g}, \operatorname{Diff}_{+}\left(S^{1}\right)\right)$. He proves the following. 
Theorem 1.6 (Bowden, see Theorem 9.5 in [1]). Let $\rho_{1}$ and $\rho_{2}: \Gamma_{g} \rightarrow \mathrm{PSL}^{(\mathrm{k})}$ be representations that lie in different connected components of $\operatorname{Hom}\left(\Gamma_{g}, \mathrm{PSL}^{(\mathrm{k})}\right)$. Then they also lie in different connected components of $\operatorname{Hom}\left(\Gamma_{g}, \operatorname{Diff}_{+}\left(S^{1}\right)\right)$.

Bowden gives two proofs, one using invariants of contact structures associated to the transverse foliation on a flat circle bundle, and the other using structural stability of Anosov flows. Both proofs assume $C^{\infty}$ regularity of diffeomorphisms, although a similar strategy might work assuming only $C^{2}$. However, the question for representations into $\operatorname{Homeo}_{+}\left(S^{1}\right)$ is essentially different, and Bowden asks if his results hold in this case. Since representations with image in $\mathrm{PSL}^{(\mathrm{k})}$ and Euler number $\frac{2 g-2}{k}$ are geometric, our Theorem 1.2 gives an affirmative answer to Bowden's question.

Translated back into the language of foliations, Theorem 1.2 says that the condition of the transverse foliation on the flat circle bundle associated to a representation $\rho$ being semiconjugate to an Anosov foliation is an open condition - any foliation $C^{0}$ close to such will still be semi-conjugate to an Anosov foliation. Compare Theorem 9.5 in [1].

\subsection{Outline}

We begin with some background on rotation numbers and the work of Calegari and Walker in [3]. This leads us to a definition of the Euler number in the language of rotation numbers, the Milnor-Wood inequality, and a description of the dynamics of representations into $\operatorname{PSL}(2, \mathbb{R})$ and $\mathrm{PSL}^{(\mathrm{k})}$ with maximal Euler number.

In Section 3 we outline our proof strategy for Theorem 1.4, motivating it by giving the proof of a toy case. Section 4 consists of a detailed study of rotation numbers of products of homeomorphisms, with the algorithm of Calegari-Walker as our main tool. We focus on examples that will later play a role in the proof of Theorem 1.4.

The proof of Theorem 1.4 is carried out in Section 5. Section 5.1 is somewhat technical, and a reader interested in only the broad flavor of the proof of our main theorems may wish to skip the proofs here on a first reading. However, the reader with an interest in rotation numbers as a tool for parametrizing or studying representation spaces should find that Section 5.1 contains some interesting techniques. Section 5.2 also uses the techniques developed in Section 4 to study rotation numbers of products of homeomorphisms, but the proofs here are much quicker.

In Section 5.3 we prove a narrower form of rotation rigidity. Extending this to the general result requires an Euclidean algorithm for rotation numbers of commutators, which we develop in Section 5.4. The reader may (again) either find this "Euclidean algorithm" technique to be of independent interest, or may choose to skip it on a first reading.

Finally, in Section 6 we deduce Theorem 1.3 from Theorem 1.4 using a trick of Matsumoto, and in Section 7 we discuss semi-conjugacy and derive Theorem 1.2 from our earlier work using results of Matsumoto and Ghys. Section 8 gives evidence for (as well as a conjecture on) the sharpness of our main theorems.

\section{Acknowledgements}

The author thanks Danny Calegari for suggesting that rotation numbers might distinguish components of $\operatorname{Hom}\left(\Gamma_{g}, \mathrm{Homeo}_{+}\left(S^{1}\right)\right)$, and explaining the philosophy of rotation numbers as "trace coordinates" on representation spaces. I am also grateful to Jonathan Bowden, Benson Farb, Shigenori Matsumoto, and Alden Walker for many helpful conversations and suggestions regarding this work, and to the referee for a careful reading and thoughtful input. 


\section{Background}

\section{$2.1 \quad$ Rotation numbers}

Let $\mathrm{Homeo}_{\mathbb{Z}}(\mathbb{R})$ denote the group of orientation-preserving homeomorphisms of $\mathbb{R}$ that commute with integral translations.

Definition 2.1. Consider $S^{1}$ as $\mathbb{R} / \mathbb{Z}$, and let $x \in S^{1}$. The $\mathbb{R} / \mathbb{Z}$-valued rotation number of an element $g \in \mathrm{Homeo}_{+}\left(S^{1}\right)$ is given by

$$
\operatorname{rot}(g):=\lim _{n \rightarrow \infty} \frac{\tilde{g}^{n}(x)}{n} \bmod \mathbb{Z}
$$

where $\tilde{g}$ is any lift of $g$ to $\operatorname{Homeo}_{\mathbb{Z}}(\mathbb{R})$.

This limit always exists, and is independent of the choice of lift $\tilde{g}$ and choice of point $x \in S^{1}$. A good exposition of the basic theory can be found in [10] or [4]. One easy fact that we will make use of is that a homeomorphism has a periodic point of period $k$ if and only if it has a rotation number of the form $m / k$ for some $m \in \mathbb{Z}$.

One can define a $\mathbb{Z}$-valued lifted rotation number (often also called "translation number") for elements of $\mathrm{Homeo}_{\mathbb{Z}}(\mathbb{R})$ in the same way.

Definition 2.2. Let $\tilde{g} \in \mathrm{Homeo}_{\mathbb{Z}}(\mathbb{R})$ and $x \in \mathbb{R}$. The lifted rotation number $\tilde{\text { rot }}(\tilde{g})$ is given by

$$
\tilde{\operatorname{rot}}(\tilde{g}):=\lim _{n \rightarrow \infty} \frac{\tilde{g}^{n}(x)}{n}
$$

Again, the limit always exists, is finite, and is independent of the choice of point $x$.

\section{Lifted commutators}

The rotation number and lifted rotation number are closely related. If $g \in \mathrm{Homeo}_{+}\left(S^{1}\right)$, and $\tilde{g}$ is a lift of $g$ to $\operatorname{Homeo}_{\mathbb{Z}}(\mathbb{R})$, then $\operatorname{rot}(g) \equiv \tilde{\operatorname{rot}}(\tilde{g}) \bmod \mathbb{Z}$, and different choices of lifts of $g$ change the value of rot $(\tilde{g})$ by an integer. However, for a commutator $g=[a, b]:=a b a^{-1} b^{-1}$, the homeomorphism $[\tilde{a}, \tilde{b}] \in \operatorname{Homeo}_{\mathbb{Z}}(\mathbb{R})$ is independent of choice of lifts $\tilde{a}$ and $\tilde{b}$ of $a$ and $b$. We will henceforth use the notation $\tilde{\operatorname{rot}}[a, b]:=\tilde{\operatorname{rot}}([\tilde{a}, \tilde{b}])$ to denote its lifted rotation number, and refer to $[\tilde{a}, \tilde{b}]$ as a lifted commutator. Similarly, for a product of commutators we define $\tilde{\operatorname{rot}}\left(\prod\left[a_{i}, b_{i}\right]\right):=\tilde{\operatorname{rot}}\left(\prod\left[\tilde{a_{i}}, \tilde{b}_{i}\right]\right)$; this also independent of the choice of lifts.

Both rot and rot are continuous with respect to the uniform norms on $\operatorname{Homeo}_{+}\left(S^{1}\right)$ and $\mathrm{Homeo}_{\mathbb{Z}}(\mathbb{R})$, are invariant under (semi-)conjugacy, and are homomorphisms when restricted to cyclic subgroups (i.e. $\tilde{\operatorname{rot}}\left(g^{n}\right)=n$ rot $(g)$ ). However, rot and rot are not homomorphisms in general - in fact it is easy to produce examples of elements of $\mathrm{Homeo}_{\mathbb{Z}}(\mathbb{R})$ with lifted rotation number zero whose product has lifted rotation number 1.

In [3], Calegari and Walker develop an approach to studying the possible values of $\operatorname{rot}(w)$ for a word $w$ in the free semigroup generated by $\tilde{a}$ and $\tilde{b}$. In particular, they give an algorithm to determine the maximum value of $\operatorname{rot}(w)$ given the lifted rotation numbers of $\tilde{a}$ and $\tilde{b}$. We will use an extension of this algorithm later in Sections 4 and 5. For now, the main result that we need is the following.

Theorem 2.3 (Theorem 3.9 in [3]). Suppose that $\tilde{\operatorname{rot}}(\tilde{a})=r$ and $\tilde{\operatorname{rot}}(\tilde{b})=s$ for some $r, s \geq 0$. Then the maximum possible value of $\tilde{\operatorname{rot}}(\tilde{a} \tilde{b})$ is given by

$$
\sup _{p_{1} / q \leq r, p_{2} / q \leq s} \frac{p_{1}+p_{2}+1}{q}
$$

where $p_{i} \geq 0$ and $q>0$ are integers. 
Calegari and Walker also show that there are strong restrictions on the possible values of the lifted rotation number of a commutator. The following result will play a crucial role in our proofs.

Lemma 2.4 (Example 4.9 in [3]). Let $a, b \in$ Homeo $_{+}\left(S^{1}\right)$. The following hold.

i) If $\operatorname{rot}(a) \notin \mathbb{Q}$ or $\operatorname{rot}(b) \notin \mathbb{Q}$, then $\tilde{\operatorname{rot}}[a, b]=0$.

ii) If $\operatorname{rot}(a)=p / q$ or $\operatorname{rot}(b)=p / q$, where $p / q \in \mathbb{Q}$ is in lowest terms, then $|\tilde{\operatorname{rot}}[a, b]| \leq 1 / q$.

We conclude this section with an elementary calculation that we will also use later.

Lemma 2.5. Let $\tilde{f}$ and $\tilde{g} \in \operatorname{Homeo}_{\mathbb{Z}}(\mathbb{R})$ satisfy $\tilde{f} \tilde{g}=T^{n}$, where $T^{n}$ denotes the translation $T^{n}(x):=x+n$, for $n \in \mathbb{Z}$. Then $\tilde{\operatorname{rot}}(\tilde{f})+\tilde{\operatorname{rot}}(\tilde{g})=n$.

Proof. If $\tilde{f}=T^{n} \circ \tilde{g}^{-1}$, then $\tilde{\operatorname{rot}}(\tilde{f})=\tilde{\operatorname{rot}}\left(T^{n} \circ \tilde{g}^{-1}\right)$. Since $T^{n}$ is a translation commuting with $\tilde{g}^{-1}$, it follows from the definition of rotation number that $\tilde{\operatorname{rot}}\left(T^{n} \circ \tilde{g}^{-1}\right)=\tilde{\operatorname{rot}}\left(T^{n}\right)+\tilde{\operatorname{rot}}\left(\tilde{g}^{-1}\right)=$ $n-\tilde{r}$ ot $(\tilde{g})$, as claimed.

\subsection{The Euler number}

Classically, the Euler number of a representation $\rho: \Gamma_{g} \rightarrow$ Homeo+ $_{+}\left(S^{1}\right)$ is defined in terms of characteristic classes - it is the result of evaluating the pullback $\rho^{*}\left(e_{\mathbb{Z}}\right) \in H^{2}\left(\Gamma_{g} ; \mathbb{Z}\right)$ of the canonical Euler class $e_{\mathbb{Z}} \in H^{2}\left(\operatorname{Homeo}_{+}\left(S^{1}\right) ; \mathbb{Z}\right)$ on the fundamental class $\left[\Gamma_{g}\right] \in H_{2}\left(\Gamma_{g} ; \mathbb{Z}\right)$. However, we will use the following alternative definition which emphasizes the relationship of the Euler number to the lifted rotation number. This idea is originally due to Milnor in [7], and is made explicit in [12].

Definition 2.6 (Euler number). Let $\left\{a_{1}, b_{1}, \ldots a_{g}, b_{g}\right\}$ be a standard set of generators for $\Gamma_{g}$, meaning that $\Gamma_{g}$ has the presentation

$$
\Gamma_{g}=\left\langle a_{1}, b_{1}, \ldots a_{g}, b_{g} \mid\left[a_{1}, b_{1}\right]\left[a_{2}, b_{2}\right] \ldots\left[a_{g}, b_{g}\right]\right\rangle .
$$

Let $\rho: \Gamma_{g} \rightarrow$ Homeo $_{+}\left(S^{1}\right)$ be a representation. We define the Euler number $\mathrm{e}(\rho)$ by

$$
\mathrm{e}(\rho):=\tilde{\operatorname{rot}}\left(\left[\rho\left(a_{1}\right), \rho\left(b_{1}\right)\right]\left[\rho\left(a_{2}\right), \rho\left(b_{2}\right)\right] \ldots\left[\rho\left(a_{g}\right), \rho\left(b_{g}\right)\right]\right) .
$$

Continuity of rot implies that e is a continuous function on $\operatorname{Hom}\left(\Gamma_{g}, G\right)$ for any subgroup $G \subset$ Homeo $_{+}\left(S^{1}\right)$. Furthermore, that $\left[a_{1}, b_{1}\right]\left[a_{2}, b_{2}\right] \ldots\left[a_{g}, b_{g}\right]$ is the identity in $\Gamma_{g}$ implies that the product of the lifted commutators $\left[\tilde{\rho}\left(a_{1}\right), \tilde{\rho}\left(b_{1}\right)\right] \ldots\left[\tilde{\rho}\left(a_{g}\right), \tilde{\rho}\left(b_{g}\right)\right]$ is an integer translation - a lift of the identity on $S^{1}$ - hence e $(\rho)$ is integer valued. It follows that $\mathrm{e}(\rho)$ is constant on connected components of $\operatorname{Hom}\left(\Gamma_{g}, G\right)$.

A remark on notation is in order. Here, and in the sequel we use the notation $\tilde{\rho}(a)$ rather than $\widetilde{\rho(a)}$ to denote a lift of $\rho(a)$ to $\operatorname{Homeo}_{\mathbb{Z}}(\mathbb{R})$ - this is not to suggest that we have lifted the representation $\rho$ to some representation $\tilde{\rho}$, but only to avoid the cumbersome notation $\widetilde{\rho(a)}$.

\section{The Milnor-Wood inequality}

The Milnor-Wood inequality implies that e takes only finitely many values on $\operatorname{Hom}\left(\Gamma_{g}, G\right)$ for any $G \subset \mathrm{Homeo}_{+}\left(S^{1}\right)$. We recall the statement here.

Theorem 2.7 (Milnor [7], Wood [12]). Let $\rho: \Gamma_{g} \rightarrow \mathrm{Homeo}_{+}\left(S^{1}\right)$ be a representation. Then $|\mathrm{e}(\rho)| \leq 2 g-2$. Furthermore, each integer value $n \in[-2 g+2,2 g-2]$ is realized as e $(\rho)$ for some representation $\rho$.

In fact, each integer $n \in[-2 g+2,2 g-2]$ is realized as $\mathrm{e}(\rho)$ for some representation $\rho: \Gamma_{g} \rightarrow \operatorname{PSL}(2, \mathbb{R})$. This gives a lower bound on the number of components of $\operatorname{Hom}\left(\Gamma_{g}, G\right)$ whenever $G$ is a subgroup of $\operatorname{Homeo}_{+}\left(S^{1}\right)$ containing $\operatorname{PSL}(2, \mathbb{R})$ - there are at least $4 g-3$ connected components, one for each value of $\mathrm{e}(\rho)$. 


\subsection{Maximal representations, cyclic covers}

The following theorem of Goldman states that representations $\rho: \Gamma_{g} \rightarrow \operatorname{PSL}(2, \mathbb{R})$ with maximal Euler number $e(\rho)= \pm(2 g-2)$ are geometric in the sense of Definition 1.1.

Theorem 2.8 (Goldman, [6]). A representation $\rho: \Gamma_{g} \rightarrow \operatorname{PSL}(2, \mathbb{R})$ has $\mathrm{e}(\rho)= \pm(2 g-2)$ if and only if it is faithful and with image a Fuchsian group.

These representations have some nice additional properties, summarized below. We leave the proofs to the reader - all are direct consequences of Theorem 2.8 and basic properties of Fuchsian groups.

Proposition 2.9. Let $\rho: \Gamma_{g} \rightarrow \operatorname{PSL}(2, \mathbb{R})$ satisfy e $(\rho)=2 g-2$, and let $\left\{a_{1}, b_{1}, \ldots a_{g}, b_{g}\right\}$ be a standard generating set for $\Gamma_{g}$. Then $\rho$ has the following properties.

i) Every homeomorphism in the image of $\rho$ has a fixed point.

ii) Each pair $\left(a_{i}, b_{i}\right)$ of generators has $\tilde{\text { rot }}\left[\rho\left(a_{i}\right), \rho\left(b_{i}\right)\right]=1$. In fact, there exists $x \in \mathbb{R}$ such that the lifted commutator satisfies $\left[\tilde{\rho}\left(a_{i}\right), \tilde{\rho}\left(b_{i}\right)\right](x)=x+1$.

iii) If $x_{i} \in S^{1}$ is the repelling fixed point for the commutator $\left[\rho\left(a_{i}\right), \rho\left(b_{i}\right)\right]$, then the cyclic order of the points $x_{i}$ on $S^{1}$ is $x_{1}<x_{2}<\ldots<x_{g}$ (i.e. cyclically lexicographic).

An analogous result holds for representations $\rho$ with Euler number $-2 g+2$. Here the cyclic order of points will be reverse lexicographic, we will have rot $\left[\rho\left(a_{i}\right), \rho\left(b_{i}\right)\right]=-1$ and the lifted commutator $\left[\tilde{\rho}\left(a_{i}\right), \tilde{\rho}\left(b_{i}\right)\right]$ will translate some point by -1 .

Representations to PSL ${ }^{(\mathrm{k})}$ with Euler number $\frac{2 g-2}{k}$ have similar dynamical properties to those listed in Proposition 2.9. These are described in the following proposition, which will serve as our starting point for the proof of Theorem 1.4.

Proposition 2.10. Let $k>0$ divide $2 g-2$ and let $\rho: \Gamma_{g} \rightarrow \mathrm{PSL}^{(\mathrm{k})}$ be a representation with Euler number $\frac{2 g-2}{k}$. Let $\left\{a_{1}, b_{1}, \ldots a_{g}, b_{g}\right\}$ be a standard generating set for $\Gamma_{g}$. The following properties hold.

i) For all $\gamma \in \Gamma_{g}$, there exists $m_{\gamma} \in \mathbb{Z}$ such that $\operatorname{rot}(\rho(\gamma))=m_{\gamma} / k$.

ii) Each pair $\left(a_{i}, b_{i}\right)$ of generators has $\operatorname{rot}\left[\rho\left(a_{i}\right), \rho\left(b_{i}\right)\right]=1 / k$ and $\tilde{\operatorname{rot}}\left[\rho\left(a_{i}\right), \rho\left(b_{i}\right)\right]=1 / k$.

iii) Let $X_{i}$ be a periodic orbit for $\left[\rho\left(a_{i}\right), \rho\left(b_{i}\right)\right]$. We may choose points $x_{i}^{0} \in X_{i}$ such that if $x_{i}^{j}=\left[\rho\left(a_{i}\right), \rho\left(b_{i}\right)\right] x_{i}^{j-1}$, then the order of the points $x_{i}^{j}$ on $S^{1}$ is cyclically lexicographic of the form

$$
x_{1}^{1}<x_{2}^{1}<\ldots<x_{g}^{1}<x_{1}^{2}<x_{2}^{2}<\ldots<x_{1}^{k}<\ldots<x_{g}^{k}
$$

Proof. Recall that $\mathrm{PSL}^{(\mathrm{k})}$ is defined via the central extension

$$
0 \rightarrow \mathbb{Z} / k \mathbb{Z} \rightarrow \mathrm{PSL}^{(\mathrm{k})} \stackrel{\pi}{\rightarrow} \operatorname{PSL}(2, \mathbb{R}) \rightarrow 1
$$

and the action of $\phi \in \mathrm{PSL}^{(\mathrm{k})}$ on $S^{1}$ is specified by

$$
\pi \circ \phi(x)=\phi\left(\operatorname{proj}_{k}(x)\right)
$$

where $\operatorname{proj}_{k}: S^{1} \rightarrow S^{1}$ is the degree- $k$ regular covering map. In particular, for each $\gamma \in \Gamma_{g}$, its image $\rho(\gamma) \in \mathrm{PSL}^{(\mathrm{k})}$ commutes with the order $k$ rotation of $S^{1}$.

Given a representation $\rho: \Gamma_{g} \rightarrow \operatorname{PSL}^{(\mathrm{k})}$ with $\mathrm{e}(\rho)=\frac{2 g-2}{k}$, consider the representation $\nu: \Gamma_{g} \rightarrow \operatorname{PSL}(2, \mathbb{R})$ defined by $\nu(\gamma)=\pi \circ \rho(\gamma)$. In other words, for each $\gamma \in \Gamma_{g}$, the homeomorphism $\rho(\gamma)$ is obtained from $\nu(\gamma)$ by choosing a particular lift of the action of $\nu(\gamma)$ on $S^{1}$ to an action on the $k$-fold cover. Since e $(\nu)=2 g-2$, Proposition 2.9 implies that $\nu(\gamma)$ has a fixed point, and so $\rho(\gamma)$ has a point whose orbit is contained in the orbit of an order $k$ rotation (the covering transformation of the $k$-fold cover). It follows that $\operatorname{rot}(\rho(\gamma))=m_{\gamma} / k$ for some integer $m_{\gamma}$. 
Now consider a pair of generators $\left(a_{i}, b_{i}\right)$. We know from Proposition 2.9 that if $\tilde{\nu}\left(a_{i}\right)$ and $\tilde{\nu}\left(b_{i}\right)$ are lifts of $\nu\left(a_{i}\right)$ and $\nu\left(b_{i}\right)$ to homeomorphisms of the infinite cyclic cover $\mathbb{R}$ of $S^{1}$, then there is some $x$ such that $\left[\tilde{\nu}\left(a_{i}\right), \tilde{\nu}\left(b_{i}\right)\right](x)=x+1$, i.e. $\left[\tilde{\nu}\left(a_{i}\right), \tilde{\nu}\left(b_{i}\right)\right]$ acts on $x$ by the covering transformation of $\mathbb{R} \rightarrow S^{1}$. It follows that if we instead take the lifts $\rho(a)$ and $\rho(b)$ to the $k$-fold cover of $S^{1}$, there will be a point $x^{\prime}$ in the cover such that $[\rho(a), \rho(b)]\left(x^{\prime}\right)$ agrees with the action of the covering transformation on $x^{\prime}$, i.e. acts on $x^{\prime}$ (and its orbit) by an order $k$ rotation. It follows that $\operatorname{rot}[\rho(a), \rho(b)]=1 / k, \tilde{\operatorname{rot}}[\rho(a), \rho(b)]=1 / k$ and that $\left[\tilde{\rho}\left(a_{i}\right), \tilde{\rho}\left(b_{i}\right)\right]$ translates any lift of $x^{\prime}$ by $1 / k$.

Finally, property iii) is an immediate consequence of Property iii) of Proposition 2.9 applied to $\nu$ and the fact that $\rho$ is the lift of $\nu$ to the $k$-fold cover.

As in Proposition 2.9, an analogous statement holds for $k<0$.

\section{Maximal PSL ${ }^{(\mathrm{k})}$ representations}

Since representations to PSL ${ }^{(\mathrm{k})}$ arise from cyclic covers, they satisfy a stronger Milnor-Wood type inequality. Namely, if $\rho$ is any representation $\Gamma_{g} \rightarrow \operatorname{PSL}^{(\mathrm{k})}$, then $\pi \circ \rho: \Gamma_{g} \rightarrow \operatorname{PSL}(2, \mathbb{R})$ has Euler number $k \mathrm{e}(\rho)$. By the Milnor-Wood inequality, we know that $|\mathrm{e}(\pi \circ \rho)| \leq 2 g-2$, so $|\mathrm{e}(\rho)| \leq \frac{2 g-2}{k}$.

We will refer to a representation $\rho: \Gamma_{g} \rightarrow \mathrm{PSL}^{(\mathrm{k})}$ with $|\mathrm{e}(\rho)|=\frac{2 g-2}{k}$ as a maximal $\mathrm{PSL}^{(\mathrm{k})}$ representation. The following proposition shows that all geometric representations are of this form.

Proposition 2.11 (Classification of geometric representations). A representation $\rho: \Gamma_{g} \rightarrow \mathrm{Homeo}_{+}\left(S^{1}\right)$ is geometric if and only if it is conjugate to a maximal $\mathrm{PSL}^{(\mathrm{k})}$ representation.

Proof. Let $\rho: \Gamma_{g} \rightarrow$ Homeo $_{+}\left(S^{1}\right)$ be a geometric representation. Then the image of $\rho$ lies in some transitive Lie subgroup of Homeo $+\left(S^{1}\right)$, and hence (up to conjugacy) lies in either in $S^{1}$ or PSL ${ }^{(\mathrm{k})}$ for some $k$ (for a proof that these are the only such Lie groups acting transitively on $S^{1}$, see Section 4 of [4]). No representation with image in $S^{1}$ can be faithful, so it follows that, after conjugacy, $\rho$ is a faithful representation to $\mathrm{PSL}^{(\mathrm{k})}$ with discrete image. Consider, as in the proof of Proposition 2.9, the homomorphism $\nu: \Gamma_{g} \rightarrow \operatorname{PSL}(2, \mathbb{R})$ defined by $\nu(\gamma)=\pi \circ \rho(\gamma)$. This also has discrete image, and since $\Gamma_{g}$ is torsion free, it must be faithful. It follows that $\mathrm{e}(\nu)= \pm(2 g-2)$, and so $\mathrm{e}(\rho)= \pm\left(\frac{2 g-2}{k}\right)$.

The converse follows immediately from our description of maximal PSL ${ }^{(\mathrm{k})}$ representations as lifts of Fuchsian representations.

Remark 2.12 (New standing assumption). From now on, it will be convenient to only work with representations such that $\mathrm{e}(\rho) \geq 0$. We claim we lose no generality in doing so. Indeed, if $\left\{a_{1}, b_{1}, \ldots a_{g}, b_{g}\right\}$ is a standard generating set for $\Gamma_{g}$, and $\rho: \Gamma_{g} \rightarrow \operatorname{Homeo}_{+}\left(S^{1}\right)$ a representation such that

$$
0>\mathrm{e}(\rho):=\tilde{\operatorname{rot}}\left(\left[\rho\left(a_{1}\right), \rho\left(b_{1}\right)\right]\left[\rho\left(a_{2}\right), \rho\left(b_{2}\right)\right] \ldots\left[\rho\left(a_{g}\right), \rho\left(b_{g}\right)\right]\right)
$$

then

$$
\begin{aligned}
0<-\mathrm{e}(\rho) & =\tilde{\operatorname{rot}}\left(\left(\left[\rho\left(a_{1}\right), \rho\left(b_{1}\right)\right]\left[\rho\left(a_{2}\right), \rho\left(b_{2}\right)\right] \ldots\left[\rho\left(a_{g}\right), \rho\left(b_{g}\right)\right]\right)^{-1}\right) \\
& =\tilde{\operatorname{rot}}\left(\left[\rho\left(b_{g}\right), \rho\left(a_{g}\right)\right]\left[\rho\left(b_{g-1}\right), \rho\left(a_{g-1}\right)\right] \ldots\left[\rho\left(b_{1}\right), \rho\left(a_{1}\right)\right]\right) .
\end{aligned}
$$

In other words, if $\sigma \in \operatorname{Aut}\left(\Gamma_{g}\right)$ is defined by permuting the generators

$$
\sigma\left(a_{i}\right)=b_{g+1-i}, \sigma\left(b_{i}\right)=a_{g+1-i},
$$


then $\rho \circ \sigma: \Gamma_{g} \rightarrow \mathrm{Homeo}_{+}\left(S^{1}\right)$ is a representation with positive Euler number. Moreover, the induced map $\rho \mapsto \rho \circ \sigma$ on $\operatorname{Hom}\left(\Gamma, \operatorname{Homeo}_{+}\left(S^{1}\right)\right)$ is clearly a homeomorphism, so permutes connected components.

Thus, for the remainder of this work it will be a standing assumption that $\mathrm{e}(\rho) \geq 0$ for all representations $\rho: \Gamma_{g} \rightarrow$ Homeo $_{+}\left(S^{1}\right)$, and maximal $\mathrm{PSL}^{(\mathrm{k})}$ representations are representations to $\operatorname{PSL}^{(\mathrm{k})}$ with $\mathrm{e}(\rho)=\frac{2 g-2}{k}$.

\section{Proof strategy for Theorem 1.4}

We continue to use the language "maximal PSL ${ }^{(\mathrm{k})}$ representation" rather than "geometric representation" as it will be convenient to refer to the number $k$ in our proofs. In these terms the statement of Theorem 1.4 is as follows

Theorem 1.4. Let $\gamma \in \Gamma_{g}$, and let $\rho_{0}$ be a maximal PSL ${ }^{(\mathrm{k})}$ representation. Then $\operatorname{rot}(\rho(\gamma))$ is constant on the component of $\operatorname{Hom}\left(\Gamma_{g}, \operatorname{Homeo}_{+}\left(S^{1}\right)\right)$ containing $\rho_{0}$.

\subsection{A toy case}

In the special case of a maximal $\mathrm{PSL}^{(2)}$ representation, and with an additional assumption on the rotation numbers of some generators, we can already prove a version of rotation number rigidity. We do this special case now to illustrate the proof strategy of Theorem 1.4 and to motivate the technical work in Sections 4 and 5.1.

Proposition 3.1 (Rotation number rigidity, toy case). Let $\rho_{0}: \Gamma_{g} \rightarrow \mathrm{SL}(2, \mathbb{R})=\mathrm{PSL}^{(2)}$ be a maximal PSL ${ }^{(2)}$ representation and let $S=\left\{a_{1}, b_{1}, \ldots a_{g}, b_{g}\right\}$ be a standard generating set for $\Gamma_{g}$. Assume additionally that that $\operatorname{rot}\left(\rho_{0}\left(a_{i}\right)\right)=1 / 2$ for all $i$. Then $\operatorname{rot}\left(\rho\left(a_{i}\right)\right)$ is constant on the component containing $\rho_{0}$.

Proof. Let $\rho_{0}$ be a maximal PSL ${ }^{(2)}$ representation as in the statement of the Proposition. By Proposition 2.10, rot $\left[\rho_{0}\left(a_{i}\right), \rho_{0}\left(b_{i}\right)\right]=1 / 2$ for all $i \in\{1,2, \ldots, g\}$. Suppose for contradiction that for some $j, \operatorname{rot}\left(\rho\left(a_{j}\right)\right)$ is not constant on the component of $\operatorname{Hom}\left(\Gamma_{g}, \operatorname{Homeo}_{+}\left(S^{1}\right)\right)$ containing $\rho_{0}$. By continuity of rot, there exists a representation $\rho$ in this component such that

i) for all $i \in\{1,2, \ldots, g\}$, we have $\operatorname{rot}\left(\rho\left(a_{i}\right)\right) \neq 0$, and

ii) there exists $j$ such that $\operatorname{rot}\left(\rho\left(a_{j}\right)\right)$ is either irrational or of the form $p / q$ with $q>2$.

It follows by Lemma 2.4 that $\operatorname{rot}\left[\rho\left(a_{i}\right), \rho\left(b_{i}\right)\right] \leq 1 / 2$ for all $i$, and $\operatorname{rot}\left[\rho\left(a_{j}\right), \rho\left(b_{j}\right)\right]<1 / 2$. For simplicity, assume that $j=g$. (This is no loss of generality as performing a cyclic permutation of the generators will not affect our proof.)

We estimate the Euler number of $\rho$ using the following lemma.

Lemma 3.2. Suppose that $\rho: \Gamma_{g} \rightarrow$ Homeo $_{+}\left(S^{1}\right)$ satisfies $\tilde{\text { rot }}\left[\rho\left(a_{i}\right), \rho\left(b_{i}\right)\right] \leq 1 / 2$ for each $i \in\{1,2, \ldots, g-1\}$. Then

$$
\tilde{\operatorname{rot}}\left(\left[\rho\left(a_{1}\right), \rho\left(b_{1}\right)\right] \ldots\left[\rho\left(a_{g-1}\right), \rho\left(b_{g-1}\right)\right]\right) \leq \frac{2 g-3}{2} .
$$

Moreover, if equality holds in $(2)$, then $\operatorname{rot}\left[\rho\left(a_{i}\right), \rho\left(b_{i}\right)\right]=1 / 2$ for all $i$.

Proof of Lemma 3.2. Let $\rho$ satisfy $\operatorname{rot}\left[\rho\left(a_{i}\right), \rho\left(b_{i}\right)\right] \leq 1 / 2$ for all $i \in\{1,2, \ldots, g-1\}$. Inductively, assume that $\tilde{r} o t\left(\left[a_{1}, b_{1}\right] \ldots\left[a_{n}, b_{n}\right]\right) \leq \frac{2 n-1}{2}$ with equality implying that $\tilde{\operatorname{rot}}\left[\rho\left(a_{i}\right), \rho\left(b_{i}\right)\right]=1 / 2$ for all $i \in\{1,2, \ldots n\}$. The base case $n=1$ is covered by Lemma 2.4. Define

$$
\begin{aligned}
& \tilde{f}:=\left[\tilde{a}_{1}, \tilde{b}_{1}\right] \ldots\left[\tilde{a}_{n}, \tilde{b}_{n}\right] \text { and } \\
& \tilde{g}:=\left[\tilde{a}_{n+1}, \tilde{b}_{n+1}\right]
\end{aligned}
$$


and apply Theorem 2.3 to $\tilde{f} \tilde{g}$. The conclusion of the theorem states that

$$
\tilde{\operatorname{rot}}(\tilde{f} \tilde{g})=\tilde{\operatorname{rot}}\left(\left[a_{1}, b_{1}\right] \ldots\left[a_{n+1}, b_{n+1}\right]\right) \leq \frac{2 n-1+1+1}{2}=\frac{2 n+1}{2}
$$

with equality only if $\tilde{\operatorname{rot}}(\tilde{f})=\frac{2 n-1}{2}$ and $\tilde{\operatorname{rot}}(\tilde{g})=\tilde{\operatorname{rot}}\left(\left[a_{n+1}, b_{n+1}\right]\right)=1 / 2$, completing the inductive step.

Returning to the proof of Proposition 3.1, by definition of Euler number we have

$$
\left[\tilde{\rho}\left(a_{1}\right), \tilde{\rho}\left(b_{1}\right)\right] \ldots\left[\tilde{\rho}\left(a_{g}\right), \tilde{\rho}\left(b_{g}\right)\right]=T^{\mathrm{e}(\rho)} .
$$

Lemma 2.5 implies that

$$
\tilde{\operatorname{rot}}\left(\left[\tilde{\rho}\left(a_{1}\right), \tilde{\rho}\left(b_{1}\right)\right] \ldots\left[\tilde{\rho}\left(a_{g-1}\right), \tilde{\rho}\left(b_{g-1}\right)\right]\right)+\tilde{\operatorname{rot}}\left[\tilde{\rho}\left(a_{g}\right), \tilde{\rho}\left(b_{g}\right)\right]=\mathrm{e}(\rho)=\frac{2 g-2}{2},
$$

and by Lemma 3.2 we have $\tilde{\operatorname{rot}}\left(\left[\tilde{\rho}\left(a_{1}\right), \tilde{\rho}\left(b_{1}\right)\right] \ldots\left[\tilde{\rho}\left(a_{g-1}\right), \tilde{\rho}\left(b_{g-1}\right)\right]\right) \leq \frac{2 g-3}{2}$. It follows that $\tilde{\operatorname{rot}}\left[\tilde{\rho}\left(a_{g}\right), \tilde{\rho}\left(b_{g}\right)\right] \geq 1 / 2$. But by hypothesis, $\operatorname{rot}\left[\tilde{\rho}\left(a_{g}\right), \tilde{\rho}\left(b_{g}\right)\right]<1 / 2$, giving the desired contradiction.

\subsection{Modifications for the general case}

Our proof of Proposition 3.1 made essential use of two special assumptions. The first was that we had a standard generating set $\left\{a_{1}, b_{1}, \ldots a_{g}, b_{g}\right\}$ such that $\operatorname{rot}\left(\rho_{0}\left(a_{i}\right)\right)=1 / 2$ held for all $i$. This allowed us to conclude that a representation $\rho$ nearby to $\rho_{0}$ satisfied $\tilde{\operatorname{rot}}\left[\tilde{\rho}\left(a_{i}\right), \tilde{\rho}\left(b_{i}\right)\right] \leq 1 / 2$ for all $i$, using Lemma 2.4. Put otherwise, we needed the fact that $\rho_{0}$ was a local maximum for the function

$$
\begin{aligned}
R_{i}: \operatorname{Hom}\left(\Gamma_{g}, \operatorname{Homeo}_{+}\left(S^{1}\right)\right) & \rightarrow \mathbb{R} \\
\rho & \mapsto \tilde{\operatorname{rot}}\left[\tilde{\rho}\left(a_{i}\right), \tilde{\rho}\left(b_{i}\right)\right]
\end{aligned}
$$

for each of the pairs $a_{i}, b_{i}$ in our standard generating set. (Of course, we could have reached the same conclusion under the assumption that $\operatorname{rot}\left(\rho_{0}\left(b_{i}\right)\right)=1 / 2$, but Lemma 2.4 does not imply anything in the case where, say, $\left.\operatorname{rot}\left(\rho_{0}\left(a_{1}\right)\right)=\operatorname{rot}\left(\rho_{0}\left(b_{1}\right)\right)=0\right)$. The meat of the proof of Theorem 1.4 lies in treating the case where images of generators have rotation number zero. This is the content of Section 5.1. (Reduction to this case is via the Euclidean algorithm for commutators of diffeomorphisms, carried out in Section 5.4.)

The second key assumption in Proposition 3.1 was that our target group was PSL ${ }^{(2)}$. We used this for the estimate on lifted rotation numbers in Lemma 3.2. To modify Lemma 3.2 for representations to $\mathrm{PSL}^{(\mathrm{k})}$, we need to bound the lifted rotation number of the product of $g-1$ homeomorphisms - the lifted commutators $\left[\tilde{\rho}\left(a_{i}\right), \tilde{\rho}\left(b_{i}\right)\right]$ - each with lifted rotation number $1 / k$. Unfortunately, the naïve approach of just repeating the argument from Lemma 3.2 for these lifted commutators gives the bound

$$
\tilde{\operatorname{rot}}\left(\left[\rho\left(a_{1}\right), \rho\left(b_{1}\right)\right] \ldots\left[\rho\left(a_{g-1}\right), \rho\left(b_{g-1}\right)\right]\right) \leq g-2
$$

independent of $k$. This is not strong enough for our purposes; we will need to take a fundamentally different approach.

The groundwork required to solve these problems is the content of the next section. We will undertake a detailed study of the behavior of lifted rotation numbers of products in $\mathrm{Homeo}_{\mathbb{Z}}(\mathbb{R})$, building on the work of Calegari and Walker in [3], and placing special emphasis on examples that arise from maximal PSL ${ }^{(k)}$ representations. In Section 5 we will use these examples to first prove that $R_{i}$ does indeed have a local maximum at $\rho_{0}$ (in fact we will prove something stronger, characterizing other local maxima), and then to find a suitable replacement for Lemma 3.2. 


\section{Rotation numbers of products of homeomorphisms}

In Section 3.2 of [3], Calegari and Walker give and algorithm for computing the maximum lifted rotation number of a product $\tilde{a} \tilde{b} \in \mathrm{Homeo}_{\mathbb{Z}}(\mathbb{R})$ given the lifted rotation numbers of $\tilde{a}$ and $\tilde{b}$. (Their algorithm also works in the more general setting of arbitrary words in $\tilde{a}$ and $\tilde{b}$ - but not words with $\tilde{a}^{-1}$ and $\left.\tilde{b}^{-1}\right)$. Assuming that $\tilde{\operatorname{rot}}(\tilde{a})$ and $\tilde{\operatorname{rot}}(\tilde{b})$ are rational, the algorithm takes as input the combinatorial structure of periodic orbits for $a$ and $b$ on $S^{1}$ (where $a$ and $b$ are the images of $\tilde{a}$ and $\tilde{b}$ under the surjection $\mathrm{Homeo}_{\mathbb{Z}}(\mathbb{R}) \rightarrow \mathrm{Homeo}_{+}\left(S^{1}\right)$ ), and as output gives the maximum possible value of $\tilde{\text { rot }}(\tilde{a} \tilde{b})$, given that combinatorial structure.

Calegari and Walker's algorithm readily generalizes to words in a larger alphabet, and we will use this generalization to prove Theorem 1.4. We find it convenient to describe the algorithm using slightly different language than that in [3]; as this will also let us treat the case (not examined in [3]) where periodic orbits of two homeomorphisms intersect nontrivially.

\subsection{The Calegari-Walker algorithm}

Let $c_{1}, c_{2}, \ldots, c_{n}$ be elements of $\mathrm{Homeo}_{+}\left(S^{1}\right)$ and let $\tilde{c}_{1}, \ldots \tilde{c}_{n}$ be lifts to Homeo $\mathbb{Z}(\mathbb{R})$. Assume that $\tilde{\operatorname{rot}}\left(c_{i}\right)=p_{i} / q_{i}$ for some integers $p_{i} \geq 0$ and $q_{i}>0$.

Let $X_{i} \subset S^{1}$ be a periodic orbit for $c_{i}$, and let $\tilde{X}_{i} \subset \mathbb{R}$ be the pre-image of $X_{i}$ under the projection $\mathbb{R} \rightarrow \mathbb{R} / \mathbb{Z}=S^{1}$. If $\operatorname{rot}\left(c_{i}\right)=0$, then one may take $X_{i}$ to be any finite subset of the set of fixed points fix $\left(c_{i}\right)$. Choose a point $x_{i}^{0} \in \tilde{X}_{i}$, and enumerate the points of $\tilde{X}_{i}$ by labeling them $x_{i}^{j}$ in increasing order, i.e. with $x_{i}^{j}<x_{i}^{j+1}$. Let $\mathbf{X}:=\left\{x_{i}^{j}: 1 \leq i \leq n, j \in \mathbb{Z}\right\} \subset \mathbb{R}$. We will define a dynamical system with orbit space $\mathbf{X}$ that encodes the "maximum distance $\tilde{c}_{i}$ can translate points to the right."

This system is generated by the $c_{i}$ acting on $\mathbf{X}$ as follows. There is a natural left-to-right order on the points $x_{i}^{j} \in \mathbf{X}$ induced by their order as a subset of $\mathbb{R}$. Each $c_{i}$ acts by moving to the right, skipping over $p_{i}$ points of $\tilde{X}_{i}$ (counting the one we start on, if we start on a point of $\left.\tilde{X}_{i}\right)$ and landing on the $\left(p_{i}+1\right)$ th. See Example 4.2 for a helpful illustration. We use $c_{i} \cdot x_{k}^{j}$ to denote the action of $c_{i}$ on a point $x_{k}^{j} \in \mathbf{X}$. Note that the action of $c_{i}$ is not in any sense an action by a homeomorphism of $\mathbf{X}$. When we want to consider $\tilde{c}_{i}$ as a homeomorphism of $\mathbb{R}$, we will use the notation $\tilde{c}_{i}(x)$.

Say that an orbit of this dynamical system is an $\frac{l}{m}$-periodic orbit for a word $w$ in the alphabet $\left\{c_{1}, c_{2}, \ldots, c_{n}\right\}$ if there exists a point $x_{i}^{j} \in \mathbf{X}$ such that

$$
w^{m} \cdot x_{i}^{j}=x_{i}^{j}+l, \text { for some } l \in \mathbb{Z} .
$$

We claim that periodic points compute the maximum possible rotation number of the homeomorphism $w\left(\tilde{c}_{1} \ldots \tilde{c}_{n}\right)$. Precisely, we have the following.

Proposition 4.1. Suppose that $w=w\left(c_{1}, c_{2}, \ldots c_{n}\right)$ is $\frac{l}{m}$-periodic. The following hold.

1. $\tilde{\operatorname{rot}}\left(w\left(\tilde{c}_{1}, \ldots \tilde{c}_{n}\right)\right) \leq \frac{l}{m}$.

2. Each homeomorphism $\tilde{c}_{i}$ can be deformed to a homeomorphism $\tilde{d}_{i} \in \mathrm{Homeo}_{\mathbb{Z}}(\mathbb{R})$ such that $\tilde{\operatorname{rot}}\left(w\left(\tilde{d}_{1}, \ldots \tilde{d}_{n}\right)\right)=\frac{l}{m}$. Moreover, the deformations can be carried out along a path of homeomorphisms preserving the lifted periodic orbits $\tilde{X}_{i}$ pointwise.

Proof. This follows from the work in Section 3.2 of [3]; we sketch the proof here for the convenience of the reader and to shed light on the meaning of the dynamical system in the algorithm. To begin, we elaborate on how the dynamical system "computes the maximum possible rotation number". In fact, what the action of $\tilde{c}_{i}$ on $\mathbf{X}$ captures is the supremal distance the homeomoprhism $\tilde{c}_{i}$ (acting on $\mathbb{R}$ ) can translate a point to the right - that $\tilde{X}_{i}$ is a lift of a periodic orbit implies that for any $y<x_{i}^{j}$ we have $\tilde{c}_{i}(y)<x_{i}^{j+p_{i}}$. This is encoded in the dynamical system as $c_{i}$ "skipping over" $p_{i}$ points of $\tilde{X}_{i}$ and landing on the $\left(p_{i}+1\right)$ th. 
Thus, that $w\left(c_{1}, c_{2}, \ldots c_{n}\right)^{m} \cdot x_{i}^{j}=x_{i}^{j}+l \in \mathbf{X}$ implies that $w\left(\tilde{c}_{1}, \tilde{c}_{2}, \ldots \tilde{c}_{n}\right) \in$ Homeo $_{\mathbb{Z}}(\mathbb{R})$ satisfies $w\left(\tilde{c}_{1}, \tilde{c}_{2}, \ldots \tilde{c}_{n}\right)^{m}\left(x_{i}^{j}\right) \leq x_{i}^{j}+l$. If $x_{i}^{j}$ is an $\frac{l}{m}$-periodic point for $w$, we have

$$
w\left(\tilde{c}_{1}, \tilde{c}_{2}, \ldots \tilde{c}_{n}\right)^{m}\left(x_{i}^{j}\right) \leq x_{i}^{j}+l
$$

and using the fact that $w\left(\tilde{c}_{1}, \tilde{c}_{2}, \ldots \tilde{c}_{n}\right)$ commutes with integer translations, this implies that

$$
w\left(\tilde{c}_{1}, \tilde{c}_{2}, \ldots \tilde{c}_{n}\right)^{k m}\left(x_{i}^{j}\right) \leq x_{i}^{j}+k l
$$

for all $k$. Hence, $\tilde{\operatorname{rot}}\left(w\left(\tilde{c}_{1}, \tilde{c}_{2}, \ldots \tilde{c}_{n}\right)\right) \leq l / m$.

We will not use the second part of Proposition 4.1 in the sequel, so our proof sketch will be brief. Choose a small $\epsilon>0$ and continuously deform the action of $\tilde{c}_{i}$ on $\mathbb{R}$, preserving its action on $\tilde{X}_{i}$, to get a homeomorphism $\tilde{d}_{i}$ that contracts each interval $\left(x_{i}^{j-1}+\epsilon, x_{i}^{j}\right]$ into $\left(x_{i}^{j+p_{i}}-\epsilon, x_{i}^{j+p_{i}}\right]$, and maps $x_{i}^{j}$ to $x_{i}^{j+p_{i}}$. It is clear that this can be done equivariantly with respect to integer translations (i.e. through a path in $\operatorname{Homeo}_{\mathbb{Z}}(\mathbb{R})$ ), and that the rotation number, which can be read off of the action of $\tilde{c}_{i}$ on $\tilde{X}_{i}$, remains constant. If $\epsilon$ is chosen close to zero, the action of $\tilde{d}_{i}$ on $\mathbb{R}$ will approximate the action of $d_{i}$ (and hence of $c_{i}$ ) on $\mathbf{X}$ given by the dynamical system described in our algorithm.

In particular, $w\left(\tilde{d}_{1}, \ldots \tilde{d}_{n}\right)^{m}\left(x_{i}^{j}\right)$ will be close to $x_{i}^{j}+l$. The fact that $\tilde{d}_{i}$ contracts the interval $\left(x_{i}^{j}+\epsilon, x_{i}^{j+1}\right]$ into $\left(x_{i}^{j+1}-\epsilon, x_{i}^{j+1}\right]$ further implies that $\left(\tilde{d}_{1} \ldots \tilde{d}_{n}\right)^{k m}\left(x_{i}^{j}\right)$ approaches $x_{i}^{j}+k l$ as $k \rightarrow \infty$. It follows from the definition of lifted rotation number that $\operatorname{rot}\left(w\left(\tilde{d}_{1}, \ldots \tilde{d}_{n}\right)\right)=l / m$.

Let us illustrate the algorithm with an example.

Example 4.2. Let $c_{1}, c_{2}, c_{3} \in \operatorname{Homeo}_{+}\left(S^{1}\right)$ satisfy $\operatorname{rot}\left(c_{i}\right)=1 / 2$ for $i=1,2,3$, and let $\tilde{c}_{i}$ be a lift with $\operatorname{rot}\left(\tilde{c}_{i}\right)=1 / 2$. Suppose the points $x_{i}^{j}$ have the following (lexicographic) order

$$
\ldots x_{1}^{j}<x_{2}^{j}<x_{3}^{j}<x_{1}^{j+1}<x_{2}^{j+1} \ldots
$$

We use the algorithm to produce an upper bound for $\tilde{\operatorname{rot}}\left(\tilde{c}_{1} \tilde{c}_{2} \tilde{c}_{3}\right)$.

Since $\operatorname{rot}\left(c_{i}\right)=1 / 2$, we have $x_{i}^{j+2 l}=x_{i}^{j}+l$ for all $i$ and $j$ and any $l \in \mathbb{Z}$. The following diagram depicts a 5/2-periodic orbit of $w=c_{1} c_{2} c_{3}$ acting on $\mathbf{X}$.

$$
x_{1}^{0} \stackrel{c_{3}}{\longmapsto} x_{3}^{1} \stackrel{c_{2}}{\longmapsto} x_{2}^{3} \stackrel{c_{1}}{\longmapsto} x_{1}^{5} \stackrel{c_{3}}{\longmapsto} x_{3}^{6} \stackrel{c_{2}}{\longmapsto} x_{2}^{8} \stackrel{c_{1}}{\longmapsto} x_{1}^{10}=x_{1}^{0}+5
$$

Hence, by Proposition 4.1, $\operatorname{rot}\left(\tilde{c}_{1} \tilde{c}_{2} \tilde{c}_{3}\right) \leq 5 / 2$.

The following example is a mild generalization of Example 4.2. It will play an important role in Section 6.

Example 4.3. Let $c_{1}, c_{2}, \ldots, c_{n} \in \operatorname{Homeo}_{+}\left(S^{1}\right)$ satisfy $\operatorname{rot}\left(c_{i}\right)=1 / k$, and let $\tilde{c}_{i}$ be the lift of $c_{i}$ to $\operatorname{Homeo}_{\mathbb{Z}}(\mathbb{R})$ with $\tilde{\operatorname{rot}}\left(\tilde{c}_{i}\right)=1 / k$. Suppose there are lifts $\tilde{X}_{i}=\left\{x_{i}^{j}\right\}$ of periodic orbits with (lexicographic) order

$$
\ldots x_{1}^{j}<x_{2}^{j}<\ldots<x_{n}^{j}<x_{1}^{j+1} \ldots
$$

We use the Calegari-Walker algorithm to show that $\tilde{\text { rot }}\left(\tilde{c}_{1} \tilde{c}_{2} \ldots \tilde{c}_{n}\right) \leq \frac{2 n-1}{k}$.

As in Example 4.2, since $\operatorname{rot}\left(c_{i}\right)=1 / k$, we have $x_{i}^{j+k l}=x_{i}^{j}+l$ for all $i$ and $j$ and any $l \in \mathbb{Z}$. We compute an orbit for $c_{1} c_{2} \ldots c_{n}$, starting with $x_{1}^{0}$.

$$
x_{1}^{0} \stackrel{c_{n}}{\longmapsto} x_{n}^{1} \stackrel{c_{n-1}}{\longmapsto} x_{n-1}^{3} \stackrel{c_{n-2}}{\longmapsto} x_{n-2}^{5} \longmapsto \ldots \stackrel{c_{1}}{\longmapsto} x_{1}^{2 n-1} \stackrel{c_{n}}{\longmapsto} x_{n}^{2 n} \stackrel{c_{n-1}}{\longmapsto} x_{n-1}^{2 n+2} \ldots
$$

In general, we have $c_{1} c_{2} \ldots c_{n} \cdot x_{1}^{j}=x_{1}^{j+2 n-1}$. After iterating this $k$ times, we have

$$
\left(c_{1} c_{2} \ldots c_{n}\right)^{k} \cdot x_{1}^{0}=x_{1}^{k(2 n-1)}=x_{1}^{0}+2 n-1
$$

This gives a $\frac{2 n-1}{k}$-periodic orbit, and we conclude that $\tilde{\operatorname{rot}}\left(\tilde{c}_{1} \tilde{c}_{2} \ldots \tilde{c}_{n}\right) \leq \frac{2 n-1}{k}$.

Note that the set-up of Example 4.3 mirrors the set-up of a maximal PSL ${ }^{(\mathrm{k})}$ representation as described in Proposition 2.10, with $c_{i}=\left[\rho\left(a_{i}\right), \rho\left(b_{i}\right)\right]$ and $\tilde{c}_{i}$ the lifted commutator $\left[\tilde{\rho}\left(a_{i}\right), \tilde{\rho}\left(b_{i}\right)\right]$. 


\subsection{Variations on Example 4.3}

We now work through two variants of Example 4.3 in which $\tilde{r}_{0}\left(\tilde{c}_{1} \tilde{c}_{2} \ldots \tilde{c}_{n}\right)$ fails to attain its maximal value of $\frac{2 n-1}{k}$. These will be used in the proof of Theorem 1.4 when we consider deformations of maximal $\mathrm{PSL}^{(\mathrm{k})}$ representations in $\operatorname{Hom}\left(\Gamma_{g}, \operatorname{Homeo}_{+}\left(S^{1}\right)\right)$.

Example 4.4 (Deforming the combinatorial structure). Let $c_{1}, c_{2}, \ldots c_{n} \in \mathrm{Homeo}_{+}\left(S^{1}\right)$ satisfy $\operatorname{rot}\left(c_{i}\right)=1 / k$, and let $\tilde{c}_{i}$ be the lift of $c_{i}$ to $\operatorname{Homeo}_{\mathbb{Z}}(\mathbb{R})$ with $\tilde{\text { rot }}\left(\tilde{c}_{i}\right)=1 / k$. Suppose for each $i$ there is a lift $\tilde{X}_{i}=\left\{x_{i}^{j}\right\}$ of a periodic orbit for $c_{i}$, with the ordering

$$
\ldots x_{1}^{j} \leq x_{2}^{j} \leq \ldots \leq x_{n}^{j} \leq x_{1}^{j+1} \ldots
$$

and at least one instance of equality, say $x_{1}^{0}=x_{2}^{0}$. We will show that $\tilde{\operatorname{rot}}\left(\tilde{c}_{1} \tilde{c}_{2} \ldots \tilde{c}_{n}\right)<\frac{2 n-1}{k}$ under the additional assumption that $2 n-1$ and $k$ are relatively prime.

First, note that $x_{1}^{0}=x_{2}^{0}$ implies that $x_{1}^{d k}=x_{2}^{d k}$ for all $d \in \mathbb{Z}$. Now for any instance where $x_{i}^{j}=x_{i+1}^{j}$ (call this a double point) observe that the dynamical system exhibits the following behavior

$$
\stackrel{c_{i+1}}{\longmapsto} x_{i+1}^{j}=x_{i}^{j} \stackrel{c_{i}}{\longmapsto} x_{i}^{j+1} \stackrel{c_{i-1}}{\longmapsto} \ldots
$$

whereas in the case where $x_{i}^{j}<x_{i+1}^{j}$ we have

$$
\stackrel{c_{i+1}}{\longmapsto} x_{i+1}^{j} \stackrel{c_{i}}{\longmapsto} x_{i}^{j+2} \stackrel{c_{i-1}}{\longmapsto} \ldots
$$

Informally speaking, double points "decrease the distance that $c_{i}$ travels to the right."

Let $w=c_{1} c_{2} \ldots c_{n}$ and consider an orbit of the action of $w$ on $\mathbf{X}$. We claim that for any initial point $x_{1}^{j}$, we have $w^{k}\left(x_{1}^{j}\right) \cdot=x_{1}^{j+s}$ for some $s<k(2 n-1)$. Indeed, the computation in Example 4.3 together with the observation that "double points decrease distance travelled to the right" shows that $s \leq k(2 n-1)$, with equality only in the case where no double points are hit along the way. In the case where no double points are hit, the orbit computation of Example 4.3 holds, and we have

$$
x_{1}^{j} \stackrel{w}{\longmapsto} x_{1}^{j+2 n-1} \stackrel{w}{\longmapsto} x_{1}^{j+2(2 n-1)} \stackrel{w}{\longmapsto} \ldots \stackrel{w}{\longmapsto} x_{i}^{j+k(2 n-1)}
$$

However, that $2 n-1$ and $k$ are relatively prime implies that $j+d(2 n-1)$ is divisible by $k$ for some $d \in\{0,1, \ldots k\}$, and by assumption this is a double point! Thus, equality cannot hold in the inequality $s \leq k(2 n-1)$.

Choose $m$ such that $k$ divides $s m$. Then $w^{k m} \cdot\left(x_{1}^{0}\right)=x_{1}^{s m}=x_{1}^{0}+s m / k$. This gives a $\frac{s m / k}{k m}=\frac{s / k}{k}$-periodic orbit for $w$, so

$$
\tilde{\operatorname{rot}}\left(w\left(\tilde{c}_{1} \tilde{c}_{2} \ldots \tilde{c}_{n}\right)\right) \leq \frac{s / k}{k}<\frac{2 n-1}{k}
$$

Remark 4.5. The reader may find it instructive to apply the algorithm in a simple case to see what fails in example 4.4 when $k$ and $n-1$ are not relatively prime; we suggest $k=3, n=4$ and $x_{1}^{3 d}=x_{2}^{3 d}$ the only instances of double points.

\section{A condition for maximality}

The Calegari-Walker algorithm produces an upper bound for the value of $\tilde{\text { rot }}\left(w\left(\tilde{c}_{1}, \ldots \tilde{c}_{n}\right)\right)$, given some data on the homeomorphisms $\tilde{c}_{i}$. It is interesting to ask under what further conditions on $\tilde{c}_{i}$ this maximum is achieved. As motivation, note that it is possible to have homeomorphisms $\tilde{c}_{i}$ satisfying the conditions in Example 4.3, but with $\tilde{\operatorname{rot}}\left(\tilde{c}_{1} \ldots \tilde{c}_{n}\right)=\frac{n}{k}$ rather than the maximum value $\frac{2 n-1}{k}$ given by the algorithm. Indeed, this will occur if we take each $\tilde{c}_{i}$ to be the translation $x \mapsto x+\frac{1}{k}$, a lift of an order $k$ rigid rotation of $S^{1}$. Appropriate choice of lifted periodic orbits will satisfy the condition on the points $x_{i}^{j}$ described in the example. 
Our next proposition (4.6) gives a condition for homeomorphisms $\tilde{c}_{i}$ as in Example 4.3 to satisfy $\operatorname{rot}\left(\tilde{c}_{1}, \ldots \tilde{c}_{n}\right)=\frac{2 n-1}{k}$, i.e. for the lifted rotation number to attain its maximal value. This proposition and its corollaries will also play an important role in the proof of Theorem 1.4.

In what follows, when we say "suppose $c_{1}, c_{2}, \ldots c_{n} \in \operatorname{Homeo}_{+}\left(S^{1}\right)$ are as in Example 4.3" we mean that $\operatorname{rot}\left(c_{i}\right)=1 / k$ for all $i$, that we have chosen lifts $\tilde{c}_{i} \in \mathrm{Homeo}_{\mathbb{Z}}(\mathbb{R})$ such that $\tilde{\operatorname{rot}}\left(\tilde{c}_{i}\right)=1 / k$, and that we have lifts $\tilde{X}_{i}=\left\{x_{i}^{j}\right\}$ of periodic orbits for the $c_{i}$ with ordering

$$
\ldots x_{1}^{j}<x_{2}^{j}<\ldots<x_{n}^{j}<x_{1}^{j+1} \ldots
$$

Proposition 4.6. Suppose $c_{1}, c_{2}, \ldots c_{n} \in \mathrm{Homeo}_{+}\left(S^{1}\right)$ are as in Example 4.3, and assume $k$ and $2 n-1$ are relatively prime. If $\tilde{\operatorname{rot}}\left(\tilde{c}_{1} \tilde{c}_{2} \ldots \tilde{c}_{n}\right)$ attains its maximal value of $\frac{2 n-1}{k}$, then for all $j \in \mathbb{Z}$ we have

$$
\left\{\begin{array}{l}
\tilde{c}_{1}\left(x_{2}^{j}\right)>x_{n}^{j+1} \\
\tilde{c}_{i}\left(x_{i+1}^{j}\right)>x_{i-1}^{j+2} \\
\tilde{c}_{n}\left(x_{1}^{j}\right)>x_{n-1}^{j+1}
\end{array} \text { for } i \neq 1, n\right.
$$

Proof. Note that rotation the number of a word in $\tilde{c}_{i}$ is invariant under cyclic permutations of the letters, meaning that $\tilde{\operatorname{rot}}\left(\tilde{c}_{1}, \ldots \tilde{c}_{n}\right)=\tilde{\operatorname{rot}}\left(\tilde{c}_{n} \tilde{c}_{1} \ldots \tilde{c}_{n-1}\right)$, etc. The proofs of each of the inequalities above are identical, after applying a cyclic permutation and re-indexing the $x_{i}^{j}$ appropriately. So we will give a proof of just the third inequality. We prove the contrapositive via a straightforward computation.

Suppose there exists $j$ such that $\tilde{c}_{n}\left(x_{1}^{j}\right) \leq x_{n-1}^{j+1}$. After relabeling, we may assume that $j=0$, so $\tilde{c}_{n}\left(x_{1}^{0}\right) \leq x_{n-1}^{1}$. Then

$$
\tilde{c}_{n-1} \tilde{c}_{n}\left(x_{1}^{j}\right) \leq \tilde{c}_{n-1}\left(x_{n-1}^{1}\right)=x_{n-1}^{2} .
$$

Considering the action of the $c_{i}$ on $\mathbf{X}$, we compute that

$$
\left(c_{1} c_{2} \ldots c_{n-2}\right) \cdot x_{n-1}^{2}=x_{1}^{2 n-2}
$$

hence $\left(\tilde{c}_{1} \tilde{c}_{2} \ldots \tilde{c}_{n-2}\right)\left(x_{n-1}^{2}\right) \leq x_{1}^{2 n-2}$. Combining this inequality with (4) gives

$$
\left(\tilde{c}_{1} \tilde{c}_{2} \ldots \tilde{c}_{n}\right)\left(x_{1}^{0}\right) \leq x_{1}^{2 n-2} .
$$

Using the fact that $2 n-1$ and $k$ are relatively prime, take integers $m>0$ and $l>0$ such that $m(2 n-1)-1=k l$. Note that $\frac{l}{m}=\frac{2 n-1-1 / m}{k}<\frac{2 n-1}{k}$.

The orbit computation from Example 4.3

shows that

$$
\left(c_{1} c_{2} \ldots . c_{n}\right)^{m-1} \cdot x_{1}^{2 n-2}=x_{1}^{2 n-2+(m-1)(2 n-1)}=x_{1}^{m(2 n-1)-1}=x_{1}^{k l}
$$

and so

$$
\left(\tilde{c}_{1} \tilde{c}_{2} \ldots \tilde{c}_{n}\right)^{m-1}\left(x_{1}^{2 n-2}\right) \leq x_{1}^{k l}
$$

Together with (5), this implies that

$$
\left(\tilde{c}_{1} \tilde{c}_{2} \ldots \tilde{c}_{n}\right)^{m}\left(x_{1}^{0}\right) \leq x_{1}^{k l}=x_{1}^{0}+l .
$$

This implies that $\tilde{\operatorname{rot}}\left(\tilde{c}_{1} \tilde{c}_{2} \ldots \tilde{c}_{n}\right)^{m} \leq l$, so $\tilde{\operatorname{rot}}\left(\tilde{c}_{1} \tilde{c}_{2} \ldots \tilde{c}_{n}\right) \leq \frac{l}{m}<\frac{2 n-1}{k}$, which is what we needed to show.

The next two corollaries of Proposition 4.6 give strong constraints on the location of other periodic points of the homeomorphisms $c_{i}$. 
Corollary 4.7. Let $c_{1}, c_{2}, \ldots c_{n} \in \mathrm{Homeo}_{+}\left(S^{1}\right)$ be as in Example 4.3. Assume $k$ and $2 n-1$ are relatively prime and that $\tilde{\operatorname{rot}}\left(\tilde{c}_{1} \tilde{c}_{2} \ldots \tilde{c}_{n}\right)$ attains its maximal value of $\frac{2 n-1}{k}$. Let $y_{i} \in S^{1}$ be any periodic point for $c_{i}$, then for any lift $\tilde{y}_{i}$ of $y_{i}$, there exists $j$ such that

$$
\left\{\begin{array}{l}
\tilde{y}_{1} \in\left(x_{n}^{j-1}, x_{2}^{j}\right) \\
\tilde{y}_{i} \in\left(x_{i-1}^{j}, x_{i+1}^{j}\right) \quad \text { for } i \neq 1, n \\
\tilde{y}_{n} \in\left(x_{n-1}^{j-1}, x_{1}^{j}\right)
\end{array}\right.
$$

Again, the three equations are all equivalent modulo cyclic permutations. One can interpret each as saying that any lift $\tilde{y}_{i}$ of a periodic point for $c_{i}$ is in the same connected component of $\mathbb{R} \backslash\left(\bigcup_{m \neq i} \tilde{X}_{m}\right)$ as a lifted periodic point $x_{i}^{j}$.

Proof. Again, we will just prove one of the inequalities, this time the second. Let $y$ denote $\tilde{y}_{i}$ and suppose that $y \notin\left(x_{i-1}^{j-1}, x_{i+1}^{j}\right)$ for any $j$. Then there exists $j$ such that $x_{i+1}^{j} \leq y \leq x_{i-1}^{j+1}$.

Then we have

$$
\tilde{c}_{i}(y) \geq \tilde{c}_{i}\left(x_{i+1}^{j}\right)>x_{i-1}^{j+2}
$$

with the second inequality following from Proposition 4.6. Applying $\left(\tilde{c}_{i}\right)^{k-1}$ to both sides, we have

$$
\left(\tilde{c}_{i}\right)^{k}(y)>\left(\tilde{c}_{i}\right)^{k-1}\left(x_{i-1}^{j+2}\right)=x_{i-1}^{j+k+1}=x_{i-1}^{j+1}+1 \geq y+1
$$

Furthermore, we know that $y \leq x_{i-1}^{j+1}<x_{i}^{j+1}$ and applying $\left(\tilde{c}_{i}\right)^{k}$ to both sides gives

$$
\left(\tilde{c}_{i}\right)^{k}(y)<x_{i}^{j+1}+1<y+2 .
$$

Thus, $1<\left(\tilde{c}_{i}\right)^{k}(y)-y<2$, so $\left(\tilde{c}_{i}\right)^{k}(y)-y$ is not an integer, and $y$ is not a lift of a periodic point for $c_{i}$.

Corollary 4.8. Let $c_{1}, c_{2}, \ldots c_{n} \in \mathrm{Homeo}_{+}\left(S^{1}\right)$ be as in Example 4.3 , assume $k$ and $2 n-1$ are relatively prime and that $\tilde{\operatorname{rot}}\left(\tilde{c}_{1} \tilde{c}_{2} \ldots \tilde{c}_{n}\right)$ attains its maximal value of $\frac{2 n-1}{\tilde{\kappa}}$. For each $i$, let $Y_{i}$ be any periodic orbit of $c_{i}$, and let $\tilde{Y}_{i}$ be its lift to $\mathbb{R}$. Then the points of $\tilde{Y}_{i}$ may be enumerated $y_{i}^{j}$ with (lexicographic) order

$$
\ldots y_{1}^{j}<y_{2}^{j}<\ldots<y_{n}^{j}<y_{1}^{j+1}<\ldots
$$

In other words, Corollary 4.8 state that all choices of periodic orbits for the homeomorphisms $c_{i}$ have the same combinatorial structure as the original choices $X_{i}$.

Proof. It suffices to prove that for any $i$ and any choice of periodic orbit $Y_{i}$ for $c_{i}$, the sets $X_{1}, \ldots X_{i-1}, Y_{i}, X_{i+1}, \ldots X_{n}$ have lifts that can be enumerated $\tilde{X}_{m}^{j}=\left\{x_{m}^{j}\right\}, \tilde{Y}_{i}^{j}=\left\{x_{i}^{j}\right\}$, with the ordering

$$
\ldots x_{1}^{j}<x_{2}^{j}<\ldots<x_{i-1}^{j}<y_{i}^{j}<x_{i+1}^{j}<\ldots<x_{n}^{j}<x_{1}^{j+1} \ldots
$$

So let $Y_{i}$ be a periodic orbit of $c_{i}$ with lift $\tilde{Y}_{i} \subset \mathbb{R}$, and let $y \in \tilde{Y}_{i}$. By Corollary 4.7, there exists $j$ such that $x_{i-1}^{j}<y<x_{i+1}^{j}$. Let $y=y_{i}^{j}$. We claim that $\tilde{c}_{i}\left(y_{i}^{j}\right) \in\left(x_{i-1}^{j+1}, x_{i+1}^{j+1}\right)$. Indeed, we know that $\tilde{c}_{i}\left(y_{i}^{j}\right) \in\left(x_{i-1}^{m}, x_{i+1}^{m+1}\right)$ for some $m$, and using the inequality above, we have

$$
x_{i}^{j}=\tilde{c}_{i}\left(x_{i}^{j-1}\right)<\tilde{c}_{i}\left(y_{i}^{j}\right)<\tilde{c}_{i}\left(x_{i+1}^{j}\right)<\tilde{c}_{i}\left(x_{i}^{j+1}\right)=x_{i}^{j+2}
$$

so it follows that $m=j-1$. The same argument now shows that $\left(\tilde{c}_{i}\right)^{d}\left(y_{i}^{j}\right) \in\left(x_{i-1}^{j+d}, x_{i+1}^{j+d}\right)$ for all $d \in \mathbb{Z}$. Defining $y_{i}^{j+d}:=\left(\tilde{c}_{i}\right)^{d}\left(y_{i}^{j}\right)$, we have a complete enumeration of the points of $\tilde{Y}_{i}$ satisfying the condition (6).

Remark 4.9. We conclude this section by noting (for the experts) that Proposition 4.6 should really be interpreted as a more general version of Matsumoto's Theorem 2.2 in [9] regarding "tame" elements of $\mathrm{Homeo}_{\mathbb{Z}}(\mathbb{R})$. 


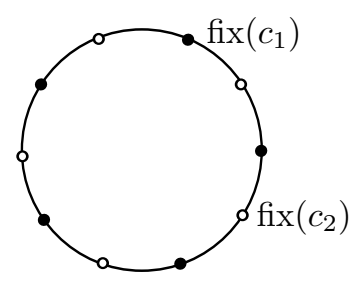

Figure 1: $k$ "alternating pairs" of fixed points for $c_{1}$ and $c_{2}$ Dark circles are fixed points of $c_{1}$, white circles are fixed points of $c_{2}$

\subsection{Homeomorphisms with fixed points}

When describing the Calegari-Walker algorithm in Section 4.1, we mentioned that if $\operatorname{rot}\left(\tilde{c}_{i}\right)=0$, then one can use any finite subset $X_{i}$ of fix $\left(c_{i}\right)$ as input for the algorithm. The following example shows that the resulting bound on $\tilde{\operatorname{rot}}\left(w\left(\tilde{c}_{1}, \ldots \tilde{c}_{n}\right)\right)$ may depend on the choice of the $X_{i}$. We will make use of this fact in the proof of Theorem 1.4.

Example 4.10. Let $c_{1}$ and $c_{2}$ be elements of $\mathrm{Homeo}_{+}\left(S^{1}\right)$ with $\operatorname{rot}\left(c_{1}\right)=\operatorname{rot}\left(c_{2}\right)=0$. Choose lifts $\tilde{c}_{i}$ of $c_{i}$ such that $\tilde{\operatorname{rot}}\left(\tilde{c}_{i}\right)=0$. Suppose there exist subsets $X_{1} \subset \operatorname{fix}\left(c_{1}\right)$ and $X_{2} \subset \operatorname{fix}\left(c_{2}\right)$ each of cardinality $k$, and such that every two points of $X_{1}$ are separated by a point of $X_{2}$ (as in Figure 1). We call such an arrangement of fixed points $k$ alternating pairs.

Then the lifts $\tilde{X}_{1}$ and $\tilde{X}_{2}$ can be ordered

$$
\ldots x_{1}^{0}<x_{2}^{0}<x_{1}^{1}<x_{2}^{1}<x_{1}^{2}<x_{2}^{2} \ldots
$$

and we have a $1 / k$-periodic orbit

$$
x_{1}^{0} \stackrel{c_{2}}{\longmapsto} x_{2}^{0} \stackrel{c_{1}}{\longmapsto} x_{1}^{1} \stackrel{c_{2}}{\longmapsto} x_{2}^{1} \stackrel{c_{1}}{\longmapsto} \ldots \stackrel{c_{2}}{\longmapsto} x_{2}^{k-1} \stackrel{c_{1}}{\longmapsto} x_{1}^{k}
$$

Hence, $\tilde{\operatorname{rot}}\left(\tilde{c}_{1} \tilde{c}_{2}\right) \leq 1 / k$.

Now we work through the algorithm with different choices of subsets of fix $\left(c_{1}\right)$ and fix $\left(c_{2}\right)$ as input. Suppose, for instance, that we choose subsets $X_{i}^{\prime} \subset \operatorname{fix}\left(c_{i}\right)$ that are singletons. Then the lifts of points $x_{i}^{j} \in \tilde{X}_{i}^{\prime}$ are again ordered

$$
\ldots x_{1}^{0}<x_{2}^{0}<x_{1}^{1}<x_{2}^{1} \ldots
$$

but now $x_{i}^{j+1}=x_{i}^{j}+1$, and

$$
x_{1}^{0} \stackrel{c_{2}}{\longmapsto} x_{2}^{0} \stackrel{c_{1}}{\longmapsto} x_{1}^{1}
$$

is a 1-periodic orbit. This gives the (weaker) bound $\operatorname{rot}\left(\tilde{c}_{1} \tilde{c}_{2}\right) \leq 1$.

The computation in Example 4.10 above illustrates the following general phenomenon.

Proposition 4.11. Let $X_{i}^{\prime} \subset X_{i}$ be finite subsets of fix $\left(c_{i}\right)$, and let $w$ be any word in $\tilde{c}_{1}, \ldots \tilde{c}_{n}$. Let $r$ be the estimate $\tilde{\text { rot }}(w) \leq r$ produced by the Calegari-Walker algorithm with inputs $X_{i}$, and let $r^{\prime}$ be the estimate $\tilde{r}$ ot $(w) \leq r^{\prime}$ produced by the algorithm with input $X_{i}^{\prime}$. Then $r \leq r^{\prime}$.

The proof is elementary and we leave it to the reader, as we will not use this proposition in the sequel. However, we do make one instructive remark which will come into play later.

Remark 4.12. Note that the inequality in Proposition 4.11 may not be strict - in other words, putting more information (a larger fixed set) into the Calegari-Walker algorithm does not always give a better estimate. For example, suppose we modify Example 4.10 by giving each of the $k$ points of $X_{1}$ and $X_{2}$ a nearby "double," as in Figure 2, to create sets $X_{1}^{\prime}$ and $X_{2}^{\prime}$ each of cardinality $2 k$. 


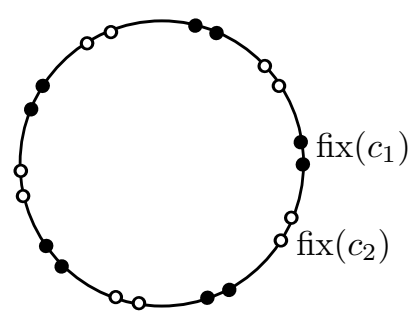

Figure 2: $k$ doubled alternating pairs of fixed points.

Dark circles are fixed points of $c_{1}$, white circles are fixed points of $c_{2}$

The lifts $\tilde{X}_{i}^{\prime}=\left\{x_{i}^{j}\right\}$ - excuse the abuse of notation- are now ordered

$$
\ldots x_{1}^{0}<x_{1}^{1}<x_{2}^{0}<x_{2}^{1}<x_{1}^{2}<x_{1}^{3}<x_{2}^{2}<x_{2}^{3} \ldots
$$

with $x_{1}^{j+2 k}=x_{1}^{j}+1$. Then

$$
x_{1}^{0} \stackrel{c_{2}}{\longmapsto} x_{2}^{0} \stackrel{c_{1}}{\longmapsto} x_{1}^{2} \stackrel{c_{2}}{\longmapsto} x_{2}^{2} \ldots \stackrel{c_{2}}{\longmapsto} x_{2}^{2 k-2} \stackrel{c_{1}}{\longmapsto} x_{1}^{2 k}
$$

is again a $1 / k$-periodic orbit, giving the estimate $\tilde{\operatorname{rot}}\left(\tilde{c}_{1} \tilde{c}_{2}\right) \leq 1 / k$.

With these examples as tools, we move on to the proof of Theorem 1.4.

\section{$5 \quad$ Proof of Theorem 1.4}

Fix a standard generating set $S=\left\{a_{1}, b_{1}, \ldots a_{g}, b_{g}\right\}$ for $\Gamma_{g}$. We will use this generating set until the end of Section 5.4.

Define $R_{i}: \operatorname{Hom}\left(\Gamma_{g}\right.$, Homeo $\left._{+}\left(S^{1}\right)\right) \rightarrow \mathbb{R}$ by

$$
R_{i}(\rho)=\tilde{\operatorname{rot}}\left[\tilde{\rho}\left(a_{i}\right), \tilde{\rho}\left(b_{i}\right)\right] .
$$

Recall from the proof outline in Section 3.2 that our first goal will be to identify show that maximal $\mathrm{PSL}^{(\mathrm{k})}$ representations are local maxima for the functions $R_{i}$. In other words, for any maximal $\mathrm{PSL}^{(\mathrm{k})}$ representation $\rho_{0}$, there is an open neighborhood $U_{i}\left(\rho_{0}\right) \subset \operatorname{Hom}\left(\Gamma_{g}, \operatorname{Homeo}_{+}\left(S^{1}\right)\right)$ such that $R_{i}(\rho) \leq R_{i}\left(\rho_{0}\right)$ holds for all $\rho \in U_{i}\left(\rho_{0}\right)$. Our strategy - which will also give us extra information that will be critical in later steps of the proof - is to define sets $N_{i}$ of representations that share some characteristics of maximal PSL ${ }^{(\mathrm{k})}$ representations. In particular, our work in Section 4 will imply that $R_{i}(\rho) \leq R_{i}\left(\rho_{0}\right)$ for all $\rho \in N_{i}$ and any maximal PSL ${ }^{(\mathrm{k})}$ representation $\rho_{0}$. We will then identify interior points of $N_{i}$ and show in particular that maximal PSL ${ }^{(\mathrm{k})}$ representations are interior points.

\subsection{Identifying local maxima of $R_{i}$}

Let $\rho_{0}$ be a maximal PSL ${ }^{(\mathrm{k})}$ representation. We will work first under the assumption that $\operatorname{rot}\left(\rho_{0}\left(a_{i}\right)\right)=0$ holds for all $i$. Then $\operatorname{rot}\left(\rho_{0}\left(a_{i}\right)^{-1}\right)=\operatorname{rot}\left(\rho_{0}\left(b a^{-1} b^{-1}\right)\right)=0$ as well. The reduction to the case that $\operatorname{rot}\left(\rho_{0}\left(a_{i}\right)\right)=0$ is carried out in Section 5.4.

Fix $i \in\{1,2, \ldots g\}$. It will be convenient to set up the following notation, consistent with the notation in Section 4.

Notation 5.1. For $\rho \in \operatorname{Hom}\left(\Gamma_{g}, \mathrm{Homeo}_{+}\left(S^{1}\right)\right)$ define

$$
c_{1}(\rho):=\rho\left(a_{i}\right), \text { and } c_{2}(\rho):=\rho\left(b_{i} a_{i}^{-1} b_{i}^{-1}\right) .
$$




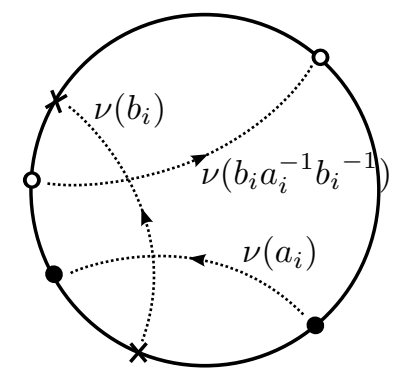

Figure 3: Dynamics of the images of standard generators under a Fuchsian representation $\nu$ Dotted arrows indicate the axes of hyperbolic elements acting on the disc

Note that, for any choice of lifts $\tilde{\rho}\left(a_{i}\right)$ and $\tilde{\rho}\left(b_{i}\right)$ of $\rho\left(a_{i}\right)$ and $\rho\left(b_{i}\right)$ to Homeo $\mathbb{Z}(\mathbb{R})$, the lifts

$$
\tilde{c}_{1}(\rho):=\tilde{\rho}\left(a_{i}\right), \text { and } \tilde{c}_{2}(\rho):=\tilde{\rho}\left(b_{i}\right) \tilde{\rho}\left(a_{i}\right)^{-1} \tilde{\rho}\left(b_{i}\right)^{-1}
$$

of $c_{1}(\rho)$ and $c_{2}(\rho)$ satisfy $\tilde{\operatorname{rot}}\left(\tilde{c}_{1}(\rho) \tilde{c}_{2}(\rho)\right)=R_{i}(\rho)$.

Our goal is to use the work of Section 4 to give bounds for $\tilde{r}\left(\tilde{c}_{1}(\rho) \tilde{c}_{2}(\rho)\right)$, given certain combinatorial data. We motivate this by examining the combinatorial structure of fix $\left(c_{1}\left(\rho_{0}\right)\right)$ and fix $\left(c_{2}\left(\rho_{0}\right)\right)$, where $\rho_{0}$ is our maximal PSL ${ }^{(\mathrm{k})}$ representation.

Structure of $\operatorname{fix}\left(c_{1}\left(\rho_{0}\right)\right)$ and $\operatorname{fix}\left(c_{2}\left(\rho_{0}\right)\right)$.

Let $X_{1}=\operatorname{fix}\left(c_{1}\left(\rho_{0}\right)\right)$ and $X_{2}=\operatorname{fix}\left(c_{2}\left(\rho_{0}\right)\right)$. We claim these sets are ordered in $S^{1}$ exactly as the "doubled alternating pairs" in Figure 2. To see this, consider first the action of a pair of standard generators $\left(a_{i}, b_{i}\right)$ under an injective, Fuchsian representation $\nu: \Gamma_{g} \rightarrow \operatorname{PSL}(2, \mathbb{R})$ with Euler number $2 g-2$. This is depicted in Figure 3.

The maximal PSL ${ }^{(\mathrm{k})}$ representation $\rho_{0}$ is a lift of such a representation $\nu$ to the $k$-fold cover of $S^{1}$, with $X_{1}$ and $X_{2}$ the lifts of fix $\left(\nu\left(a_{i}\right)\right)$ and fix $\left(\nu\left(b_{i} a_{i}^{-1} b_{i}^{-1}\right)\right)$ respectively. The fact that the points of fix $\left(\nu\left(a_{i}\right)\right)$ do not separate those of fix $\left(\nu\left(b_{i} a_{i}^{-1} b_{i}^{-1}\right)\right)$ on $S^{1}$ implies that points of $X_{1}$ and $X_{2}$ on the $k$-fold cover appear in "doubled alternating pairs" exactly as in Figure 2 from Remark 4.12.

As in Example 4.10, the lifted fixed points $\tilde{X}_{1}=\left\{x_{1}^{j}\right\}$ and $\tilde{X}_{2}=\left\{x_{2}^{j}\right\}$ may be ordered

$$
\ldots x_{1}^{0}<x_{1}^{1}<x_{2}^{0}<x_{2}^{1}<x_{1}^{2}<x_{1}^{3}<x_{2}^{2}<x_{2}^{3} \ldots
$$

We also know that $\tilde{\rho_{0}}\left(b_{i}\right)\left(\tilde{X}_{1}\right)=\tilde{X}_{2}$. Since $\tilde{\operatorname{rot}}\left(\tilde{\rho_{0}}\left(b_{i}\right)\right)=\frac{n}{k}$ for some $n$, it follows from the dynamics of the action of $\nu\left(b_{i}\right)$ in the Fuchsian case (as in Figure 3) that $\tilde{\rho}_{0}\left(b_{i}\right)\left(x_{1}^{2 j}\right)=x_{2}^{2 j+2 n-1}$ and $\tilde{\rho}_{0}\left(b_{i}\right)\left(x_{1}^{2 j+1}\right)=x_{2}^{2 j+2 n}$ for all $j$. See Figure 4 .

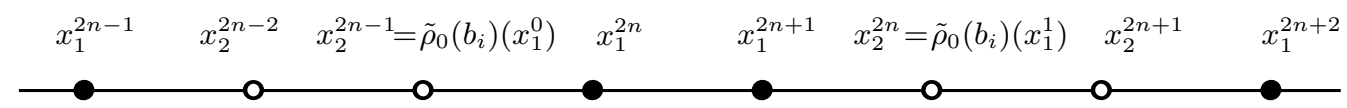

Figure 4: Order of points $x_{i}^{j}$ and $\tilde{\rho}_{0}\left(b_{i}\right) x_{i}^{j}$

\section{Representations with good fixed sets.}

For general $\rho \in \operatorname{Hom}\left(\Gamma_{g}\right.$, Homeo $\left._{+}\left(S^{1}\right)\right)$, we say that $c_{1}(\rho)$ has a good fixed set if it fixes a set of points that "look like" the set $X_{1}\left(\rho_{0}\right)$ above.

Definition 5.2. For $\rho \in \operatorname{Hom}\left(\Gamma_{g}, \operatorname{Homeo}_{+}\left(S^{1}\right)\right)$, say that $X_{1}(\rho) \subset \operatorname{fix}\left(c_{1}(\rho)\right)$ is a good fixed set for $c_{1}(\rho)=\rho\left(a_{i}\right)$ if the following hold: 
i) $X_{1}(\rho)$ is of cardinality $2 k$.

ii) If we define $X_{2}(\rho)$ by $X_{2}(\rho):=\rho\left(b_{i}\right)\left(X_{1}(\rho)\right) \subset \operatorname{fix}\left(c_{2}(\rho)\right)$, then their lifts $\tilde{X}_{1}(\rho)=\left\{x_{1}^{j}(\rho)\right\}$ and $\tilde{X}_{2}(\rho)=\left\{x_{2}^{j}(\rho)\right\} \subset \mathbb{R}$ can be indexed such that

$$
\ldots x_{1}^{0}(\rho)<x_{1}^{1}(\rho)<x_{2}^{0}(\rho)<x_{2}^{1}(\rho)<x_{1}^{2}(\rho)<x_{1}^{3}(\rho)<x_{2}^{2}(\rho)<x_{2}^{3}(\rho) \ldots
$$

iii) With the indexing giving the order above, we have

$$
\tilde{\rho}\left(b_{i}\right)\left(x_{1}^{2 j}(\rho)\right)=x_{2}^{2 j+2 n-1}(\rho) \text { and } \tilde{\rho}\left(b_{i}\right)\left(x_{1}^{2 j+1}(\rho)\right)=x_{2}^{2 j+2 n}(\rho) .
$$

Example 4.10 shows that if $\rho\left(a_{i}\right)$ has a good fixed set, then $\tilde{\operatorname{rot}}\left[\tilde{\rho}\left(a_{i}\right), \tilde{\rho}\left(b_{i}\right)\right]=R_{i}(\rho) \leq 1 / k$.

Let $N_{i}$ be the closure in $\operatorname{Hom}\left(\Gamma_{g}, \mathrm{Homeo}_{+}\left(S^{1}\right)\right)$ of the set

$$
\left\{\rho \in \operatorname{Hom}\left(\Gamma_{g}, \mathrm{Homeo}_{+}\left(S^{1}\right)\right) \mid \rho\left(a_{i}\right) \text { has a good fixed set }\right\} .
$$

Then $R_{i}(\rho) \leq 1 / k$ holds for all $\rho \in N_{i}$ as well. We claim that representations in $N_{i}$ where $R_{i}(\rho)$ attains the maximum value are interior points of $N_{i}$.

Proposition 5.3 (Interior points of $\left.N_{i}\right)$. Let $\rho \in N_{i}$ satisfy $R_{i}(\rho)=1 / k$. Then $N_{i}$ contains an open neighborhood of $\rho$ in $\operatorname{Hom}\left(\Gamma_{g}, \operatorname{Homeo}_{+}\left(S^{1}\right)\right)$.

Proof. Let $\rho \in N_{i}$ be such that $R_{i}(\rho)=1 / k$. Since $\rho \in N_{i}$, we may take a sequence of representations $\rho_{n}$ approaching $\rho$ such that each $\rho_{n}$ has a good fixed set $X_{1}\left(\rho_{n}\right) \subset \operatorname{fix}\left(c_{1}\left(\rho_{n}\right)\right)$. Since the space of $k$-tuples in $S^{1}$ is compact, after passing to a subsequence, we may assume that $X_{1}\left(\rho_{n}\right)$ converges to a $2 k$-tuple of (a priori not necessarily distinct) points $X_{1}(\rho) \subset S^{1}$. We claim that

i) $X_{1}(\rho)$ is a good fixed set for $c_{1}(\rho)$, and

ii) Any $\rho^{\prime}$ sufficiently close to $\rho$ has a good fixed set.

\section{i) Proof that $X_{1}(\rho)$ is a good fixed set}

That $\rho_{n}$ converges to $\rho$ implies that $X_{1}(\rho) \subset \operatorname{fix}\left(c_{1}(\rho)\right)$. Let $\tilde{X}_{1}\left(\rho_{n}\right) \subset \mathbb{R}$ be the set of all lifts of points in $X_{1}\left(\rho_{n}\right)$. If we choose to enumerate $X_{1}\left(\rho_{n}\right)$ so that the points $x_{1}^{0}\left(\rho_{n}\right)$ stay within a compact set, then after passing to a further subsequence we may assume that for each $j$, the sequence $x_{1}^{j}\left(\rho_{n}\right)$ converges to a point $x_{1}^{j}(\rho) \in \tilde{X}_{1}(\rho)$. Then $x_{2}^{j}\left(\rho_{n}\right)$ converges to some point $x_{2}^{j}(\rho)$. These points are ordered

$$
\ldots x_{1}^{0}(\rho) \leq x_{1}^{1}(\rho) \leq x_{2}^{0}(\rho) \leq x_{2}^{1}(\rho) \leq x_{1}^{2}(\rho) \leq x_{1}^{3}(\rho) \leq x_{2}^{2}(\rho) \leq x_{2}^{3}(\rho) \ldots
$$

with

$$
\tilde{\rho}\left(b_{i}\right)\left(x_{1}^{2 j}(\rho)\right)=x_{2}^{2 j+2 n-1}(\rho) \text {, and } \tilde{\rho}\left(b_{i}\right)\left(x_{1}^{2 j+1}(\rho)\right)=x_{2}^{2 j+2 n}(\rho) .
$$

We need only show that all the inequalities in $(7)$ are strict. This is straightforward: if $x_{1}^{j}(\rho)=x_{2}^{l}(\rho)$ for some $l$ and $j$, then $\operatorname{fix}\left(c_{1}(\rho)\right) \cap \operatorname{fix}\left(c_{2}(\rho)\right) \neq \emptyset$, so $\tilde{\operatorname{rot}}\left(\tilde{c}_{1}(\rho) \tilde{c}_{2}(\rho)\right)=0$, contradicting our assumption. Furthermore, since $x_{1}^{l}(\rho) \neq x_{2}^{j}(\rho)$ for all $l$ and $j$, it follows from Equation (8) that $x_{1}^{j}(\rho) \neq x_{1}^{l}(\rho)$ for all $l \neq j$ as well.

\section{ii) Proof that nearby representations have good fixed sets}

As the proof of this is slightly technical, we start with an easy case for motivation. Suppose that we knew that the points of $X_{1}(\rho)$ were stable fixed points - meaning that for any $\epsilon>0$, there is a neighborhood $U_{\epsilon}$ of $\rho$ in $\operatorname{Hom}\left(\Gamma_{g}, \mathrm{Homeo}_{+}\left(S^{1}\right)\right)$ such that $X_{1}(\rho)$ is contained within the $\epsilon$-neighborhood of fix $\left(c_{1}\left(\rho^{\prime}\right)\right)$ for each $\rho^{\prime} \in U_{\epsilon}$. (This is satisfied, for example, if each point $x \in X_{1}(\rho)$ is either a source or a sink for $c_{1}(\rho)$.) In this case, for each $\rho^{\prime} \in U_{\epsilon}$, we can pick a 


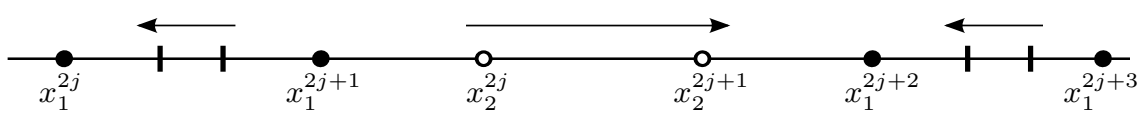

Figure 5: dynamics of $\tilde{c}_{1}(\rho)$

subset $X_{1}\left(\rho^{\prime}\right) \subset \operatorname{fix}\left(c_{1}\left(\rho^{\prime}\right)\right)$ of cardinality $2 k$ that is of Hausdorff distance at most $\epsilon$ from $X_{1}(\rho)$. Provided $\epsilon$ is sufficiently small, this will be a good fixed set for $\rho^{\prime}$.

To treat the general case, we will first do some further analysis of the dynamics of $\rho$. Our goal is to identify other "stable conditions" that imply that nearby representations $\rho^{\prime}$ have good fixed sets. To make the next few lemmas easier to read, we drop " $\rho$ " from the notation, writing $x_{i}^{j}$ for $x_{i}^{j}(\rho)$.

Lemma 5.4. $\tilde{c}_{2}(\rho)$ is strictly increasing on each interval $\left[x_{1}^{2 j}, x_{1}^{2 j+1}\right]$.

Proof. We use the fact that $R_{i}(\rho)=\tilde{\operatorname{rot}}\left(\tilde{c}_{1}(\rho) \tilde{c}_{2}(\rho)\right)=\frac{1}{k}$.

Suppose for contradiction that $\tilde{c}_{2}(\rho)$ is not strictly increasing on some $\left[x_{1}^{2 j}, x_{1}^{2 j+1}\right]$. There are three cases. First, suppose that $\tilde{c}_{2}(\rho)$ has a fixed point $y$ in $\left(x_{1}^{2 j}, x_{1}^{2 j+1}\right)$. Let $\bar{y} \in S^{1}$ be the projection of $y$ to $S^{1}=\mathbb{R} / \mathbb{Z}$ and consider the sets

$$
\begin{gathered}
Y_{2}:=X_{2}(\rho) \cup\{\bar{y}\} \subset \operatorname{fix}\left(c_{2}(\rho)\right), \text { and } \\
Y_{1}:=\rho\left(b_{i}\right)^{-1}\left(Y_{2}\right) \subset \operatorname{fix}\left(c_{1}(\rho)\right) .
\end{gathered}
$$

Then $Y_{1}$ and $Y_{2}$ contain $k+1$ alternating pairs, so by Example 4.10, rot $\left(\tilde{c}_{1}(\rho) \tilde{c}_{2}(\rho)\right) \leq \frac{1}{k+1}$. This is a contradiction.

If instead $\tilde{c}_{2}(\rho)$ fixes an endpoint of $\left[x_{1}^{2 j}, x_{1}^{2 j+1}\right]$, then $\tilde{c}_{1}(\rho)$ and $\tilde{c}_{2}(\rho)$ have a common fixed point, and so rot $\left(\tilde{c}_{1}(\rho) \tilde{c}_{2}(\rho)\right)=0$. Finally, if $\tilde{c}_{2}(\rho)$ is strictly decreasing on $\left[x_{1}^{2 j}, x_{1}^{2 j+1}\right]$, then $\tilde{c}_{1}(\rho) \tilde{c}_{2}(\rho)\left(x_{1}^{2 j+1}\right)<\tilde{c}_{1}(\rho)\left(x_{1}^{2 j+1}\right)=x_{1}^{2 j+1}$ and so $\tilde{\operatorname{rot}}\left(\tilde{c}_{1}(\rho) \tilde{c}_{2}(\rho)\right) \leq 0$, again a contradiction.

Essentially the same argument can be used to prove the following lemma (we omit the proof). Lemma 5.5. $\tilde{c}_{1}(\rho)$ is strictly increasing on each interval $\left[x_{2}^{2 j}, x_{2}^{2 j+1}\right]$.

Since $\tilde{c}_{2}(\rho)$ is strictly increasing on $\left[x_{1}^{2 j+2 n}, x_{1}^{2 j+2 n+1}\right]$, its inverse $\tilde{\rho}\left(b_{i}\right)^{-1} \tilde{\rho}\left(a_{i}\right) \tilde{\rho}\left(b_{i}\right)$ is strictly decreasing on $\left[x_{1}^{2 j+2 n}, x_{1}^{2 j+2 n+1}\right]$, and so $\tilde{c}_{1}(\rho)=\tilde{\rho}\left(a_{i}\right)$ is strictly decreasing on

$$
\tilde{\rho}\left(b_{i}\right)^{-1}\left[x_{1}^{2 j+2 n}, x_{1}^{2 j+2 n+1}\right] \subset\left(x_{1}^{2 j}, x_{1}^{2 j+1}\right) .
$$

The content of Lemmas 5.4 and 5.5 is summarized in Figure 5, with arrows indicating where $\tilde{c}_{1}(\rho)$ is increasing and decreasing.

The next two lemmas give a refinement of this picture.

Lemma 5.6. Let

$$
y^{2 j+1}=\min \left(\operatorname{fix}\left(\tilde{c}_{1}(\rho)\right) \cap\left[\tilde{\rho}\left(b_{i}\right)^{-1}\left(x_{1}^{2 j+2 n+1}\right), x_{2}^{2 j}\right]\right)
$$

and let

$$
z^{2 j+1}=\max \left(\operatorname{fix}\left(\tilde{c}_{1}(\rho)\right) \cap\left[\tilde{\rho}\left(b_{i}\right)^{-1}\left(x_{1}^{2 j+2 n+1}\right), x_{2}^{2 j}\right]\right)
$$

Then $\tilde{\rho}\left(b_{i}\right) y^{2 j+1}>z^{2 j+2 n+1}$.

The indexing of points in Lemma 5.6 is designed so that $y^{2 j+1}$ and $z^{2 j+1}$ are close to $x_{1}^{2 j+1}$. 


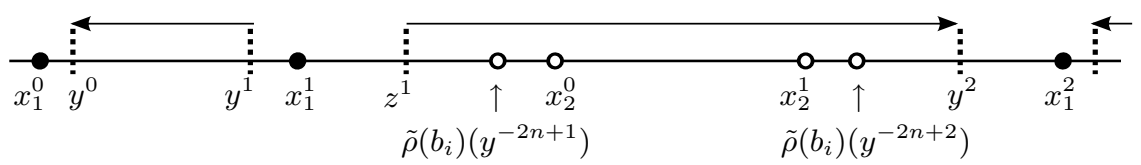

Figure 6: A more precise picture of the dynamics of $\tilde{c}_{1}(\rho)$ after Lemma 5.7

Proof. As in Lemma, 5.4, this follows from the fact that $R_{i}(\rho)=\tilde{\operatorname{rot}}\left(\tilde{c}_{1}(\rho) \tilde{c}_{2}(\rho)\right)=\frac{1}{k}$. Suppose for contradiction that $\tilde{\rho}\left(b_{i}\right) y^{2 j+1} \leq z^{2 j+2 n+1}$ holds for some $j$. If equality holds, then $\tilde{c}_{1}(\rho)$ and $\tilde{c}_{2}(\rho)$ have a common fixed point, so $R_{i}(\rho)=0$, a contradiction. Otherwise, let $\bar{y}$ denote the projection of $y^{2 j+1}$ to $S^{1}$ and $\bar{z}$ the projection of $z^{2 j+2 n+1}$, and define

$$
\begin{gathered}
Y_{1}:=X_{1}(\rho) \cup\{\bar{y}, \bar{z}\} \subset \operatorname{fix}\left(\rho\left(c_{1}\right)\right), \text { and } \\
Y_{2}:=\rho\left(b_{1}\right) Y_{1} \subset \operatorname{fix}\left(c_{2}(\rho)\right) .
\end{gathered}
$$

Because $x_{1}^{2 j+2 n+1}<\tilde{\rho}\left(b_{i}\right) y^{2 j+1}<z^{2 j+2 n+1}<x_{2}^{2 j+2 n}$, the sets $Y_{1}$ and $Y_{2}$ contain $k+1$ alternating pairs. By example 4.10, $\tilde{\operatorname{rot}}\left(\tilde{c}_{1}(\rho) \tilde{c}_{2}(\rho)\right) \leq \frac{1}{k+1}$, a contradiction.

The same type of argument also shows the following.

Lemma 5.7. Let

$$
\begin{gathered}
y^{2 j}=\max \left(\operatorname{fix}\left(\tilde{c}_{1}(\rho)\right) \cap\left[x_{2}^{2 j}, \tilde{\rho}\left(b_{i}\right)^{-1}\left(x_{1}^{2 j+2 n}\right)\right]\right) \text { and } \\
z^{2 j}=\min \left(\operatorname{fix}\left(\tilde{c}_{1}(\rho)\right) \cap\left[x_{2}^{2 j}, \tilde{\rho}\left(b_{i}\right)^{-1}\left(x_{1}^{2 j+2 n}\right)\right]\right)
\end{gathered}
$$

Then $\tilde{\rho}\left(b_{i}\right) y^{2 j}<z^{2 j+2 n}$.

Figure 6 summarizes what we now know about the dynamics of $c_{1}(\rho)$. (To avoid too many " $j$ "s in the superscripts, the figure starts with $x_{1}^{0}$ rather than the general $x_{1}^{2 j}$.)

Now we can show that any representation $\rho^{\prime}$ sufficiently close to $\rho$ has a good fixed set. It follows from Lemmas 5.6 and 5.7 that for all sufficiently small $\epsilon>0$, there exists a neighborhood $U_{\epsilon}$ of $\rho$ in $\operatorname{Hom}\left(\Gamma_{g}, \operatorname{Homeo}_{+}\left(S^{1}\right)\right)$ such that, for each $\rho^{\prime} \in U_{\epsilon}$, there are lifts $\tilde{c}_{1}\left(\rho^{\prime}\right)$ of $c_{1}\left(\rho^{\prime}\right)$ and $\tilde{\rho}^{\prime}\left(b_{i}\right)$ of $\rho^{\prime}\left(b_{i}\right)$ satisfying the following:

i) $\tilde{c}_{1}\left(\rho^{\prime}\right)(x)<x$ for $x \in\left[y^{2 j}+\epsilon, y^{2 j-1}-\epsilon\right]$,

ii) $\tilde{c}_{1}\left(\rho^{\prime}\right)(x)>x$ for $\left.x \in\left[\left(z^{2 j-1}+\epsilon\right), z^{2 j}-\epsilon\right)\right]$,

iii) $\tilde{\rho}^{\prime}\left(b_{i}\right)\left(y^{2 j+1}-\epsilon\right)>z^{2 j+2 n+1}+\epsilon$, and

iv) $\tilde{\rho}^{\prime}\left(b_{i}\right)\left(y^{2 j}+\epsilon\right)>z^{2 j+2 n}-\epsilon$, for all $j$.

We may take $\epsilon$ independent of $j$ because $\tilde{c}_{1}\left(\rho^{\prime}\right)$ and $\tilde{\rho}^{\prime}\left(b_{i}\right)$ are lifts of homeomorphisms of $S^{1}$.

It follows that $\tilde{c}_{1}\left(\rho^{\prime}\right)$ has a fixed point in each interval $\left(y^{2 j+1}-\epsilon, z^{2 j+1}+\epsilon\right)$, as well as in each interval $\left(z^{2 j}-\epsilon, y^{2 j}+\epsilon\right)$. The reader may find it helpful to refer to Figure 6. Choose a point

$$
x_{1}^{2 j+1}\left(\rho^{\prime}\right) \in \operatorname{fix}\left(\tilde{c}_{1}\left(\rho^{\prime}\right)\right) \cap\left(y^{2 j+1}-\epsilon, z^{2 j+1}+\epsilon\right),
$$

and a point

$$
x_{1}^{2 j}\left(\rho^{\prime}\right) \in \operatorname{fix}\left(\tilde{c}_{1}\left(\rho^{\prime}\right)\right) \cap\left(z^{2 j}-\epsilon, y^{2 j}+\epsilon\right)
$$

for each $j$, and do so equivariantly with respect to integer translation so that their union $\tilde{X}_{1}\left(\rho^{\prime}\right):=\left\{x_{1}^{m} \mid m \in \mathbb{Z}\right\}$ is the lift of a subset $X_{1}\left(\rho^{\prime}\right) \subset \operatorname{fix}\left(\rho^{\prime}\left(a_{i}\right)\right) \subset S^{1}$ with $\left|X_{1}\left(\rho^{\prime}\right)\right|=2 k$. Our next goal is to show that $X_{1}\left(\rho^{\prime}\right)$ is a good fixed set for $\rho^{\prime}$.

As in the definition of good fixed set, define $x_{2}^{2 j+2 n-1}\left(\rho^{\prime}\right):=\tilde{\rho}^{\prime}\left(b_{i}\right)\left(x_{1}^{2 j}\left(\rho^{\prime}\right)\right)$ and $x_{2}^{2 j+2 n}\left(\rho^{\prime}\right):=$ $\tilde{\rho}^{\prime}\left(b_{i}\right)\left(x_{1}^{2 j+1}\left(\rho^{\prime}\right)\right)$. It remains only to show that the points $x_{1}^{m}$ and $x_{2}^{m}$ are ordered as in condition ii) in the definition of good fixed set. 
By construction, $x_{1}^{m}<x_{1}^{m+1}$ holds for all $m \in \mathbb{Z}$, and therefore $x_{2}^{m}<x_{2}^{m+1}$ holds as well. Moreover, our choice of points $x_{1}^{m}$ gives, for all $j$,

$$
x_{2}^{2 j+2 n}\left(\rho^{\prime}\right)=\tilde{\rho}^{\prime}\left(b_{i}\right)\left(x_{1}^{2 j+1}\left(\rho^{\prime}\right)\right)>\tilde{\rho}^{\prime}\left(b_{i}\right)\left(y^{2 j+1}-\epsilon\right)>z^{2 j+2 n+1}+\epsilon>x_{1}^{2 j+2 n+1}\left(\rho^{\prime}\right)
$$

and similarly

$$
x_{2}^{2 j+2 n-1}\left(\rho^{\prime}\right)=\tilde{\rho}^{\prime}\left(b_{i}\right)\left(x_{1}^{2 j}\left(\rho^{\prime}\right)\right)<\tilde{\rho}^{\prime}\left(b_{i}\right)\left(y^{2 j}+\epsilon\right)<z^{2 j+2 n}-\epsilon<x_{1}^{2 j+2 n}\left(\rho^{\prime}\right) .
$$

These two inequalities together imply that

$$
\ldots x_{1}^{0}\left(\rho^{\prime}\right)<x_{1}^{1}\left(\rho^{\prime}\right)<x_{2}^{0}\left(\rho^{\prime}\right)<x_{2}^{1}\left(\rho^{\prime}\right)<x_{1}^{2}\left(\rho^{\prime}\right)<x_{1}^{3}\left(\rho^{\prime}\right)<x_{2}^{2}\left(\rho^{\prime}\right)<x_{2}^{3}\left(\rho^{\prime}\right) \ldots
$$

so $X_{1}\left(\rho^{\prime}\right)$ is a good fixed set for $\rho^{\prime}$.

This concludes the proof of Proposition 5.3.

\subsection{Rotation numbers of products of commutators}

Similar to what we did in Section 5.1, our strategy here is to define a good representation to be one that "looks like" a maximal PSL ${ }^{(\mathrm{k})}$ representation (at least on the level of the combinatorial data of some periodic points for certain elements), determine the possible values of $R(\rho)$ for "good" representations $\rho$, and then find interior points of the set of good representations. It will be convenient to introduce a little more notation.

Notation 5.8. Given any $\rho \in \operatorname{Hom}\left(\Gamma_{g}, \operatorname{Homeo}_{+}\left(S^{1}\right)\right.$, let $c_{i}(\rho)$ denote the lifted commutator $\left[\tilde{\rho}\left(a_{i}\right), \tilde{\rho}\left(b_{i}\right)\right] \in$ Homeo $_{\mathbb{Z}}(\mathbb{R})$, and let $R(\rho)$ denote the rotation number of the product $\operatorname{rot}\left(c_{1}(\rho) c_{2}(\rho) \ldots c_{g-1}(\rho)\right)$.

Definition 5.9. Say that a representation $\rho \in \operatorname{Hom}\left(\Gamma_{g}, \operatorname{Homeo}_{+}\left(S^{1}\right)\right)$ is good if the following two conditions hold.

i) $\operatorname{rot}\left(\tilde{c}_{i}(\rho)\right)=1 / k$ for all $i \in\{1,2, \ldots g-1\}$, and

ii) For each $i$, there is a periodic orbit $X_{i}(\rho)$ of $c_{i}(\rho)$ such that the lifts $\tilde{X}_{i}(\rho)$ can be ordered

$$
\ldots x_{1}^{j}(\rho)<x_{2}^{j}(\rho)<\ldots<x_{g-1}^{j}(\rho)<x_{1}^{j+1}(\rho) \ldots
$$

Let $N_{0}$ be the closure of the set of good representations in $\operatorname{Hom}\left(\Gamma_{g}, \mathrm{Homeo}_{+}\left(S^{1}\right)\right)$. Note that our maximal PSL ${ }^{(\mathrm{k})}$ representation $\rho_{0}$ is a good representation, and that for any good representation $\rho$ Example 4.3 implies that

$$
\tilde{\operatorname{rot}}\left(\tilde{c}_{1}(\rho) \tilde{c}_{2}(\rho) \ldots \tilde{c}_{g-1}(\rho)\right)=\tilde{\operatorname{rot}}\left(\left[\tilde{\rho}\left(a_{1}\right), \tilde{\rho}\left(b_{1}\right)\right] \ldots\left[\tilde{\rho}\left(a_{g-1}\right), \tilde{\rho}\left(b_{g-1}\right)\right]\right) \leq \frac{2 g-3}{2}
$$

Moreover, (9) holds not only for good representations but for all $\rho \in N_{0}$. Finally, for $i=1,2, \ldots g$ let $N_{i}$ be the set defined in Section 5.1 and define $N=\bigcap_{i=0}^{g} N_{i}$.

Proposition 5.10 (Interior points of $N$ ). Let $\rho \in N$. If $\tilde{\text { rot }}\left(\tilde{c}_{1}(\rho) \tilde{c}_{2}(\rho) \ldots \tilde{c}_{g-1}(\rho)\right)=\frac{2 g-3}{k}$ holds, then $\rho$ is an interior point of $S$.

Proof. Suppose that $\rho \in N$ satisfies $\tilde{\operatorname{rot}}\left(\tilde{c}_{1}(\rho) \tilde{c}_{2}(\rho) \ldots \tilde{c}_{g-1}(\rho)\right)=\frac{2 g-3}{2}$. Let $\rho_{n}$ be a sequence of representations in $\bigcap_{i=0}^{g} N_{i}$ approaching $\rho$. Then $\rho_{n} \in N_{0}$, so we may choose periodic orbits $X_{i}\left(\rho_{n}\right)$ 
for $c_{i}\left(\rho_{n}\right)$ as in the definition of "good". After passing to a subsequence, we may assume that each $X_{i}\left(\rho_{n}\right)$ converges to a $k$-tuple of points $X_{i}(\rho)$ with lifts ordered

$$
\ldots x_{1}^{j}(\rho) \leq x_{2}^{j}(\rho) \leq \ldots \leq x_{n}^{j}(\rho) \leq x_{1}^{j+1}(\rho) \ldots
$$

If equality holds in any of these inequalities, then $\tilde{\operatorname{rot}}\left(\tilde{c}_{1}(\rho) \tilde{c}_{2}(\rho) \ldots \tilde{c}_{g-1}(\rho)\right)<\frac{2 g-3}{2}$ holds by Example 4.4. This contradicts our assumption, so all points $x_{i}^{j}(\rho)$ must be distinct.

Since $\tilde{\operatorname{rot}}\left(\tilde{c}_{i}(\rho)\right)=1 / k$, by Proposition 5.3 there is an open neighborhood $U_{i}$ of $\rho$ in $\operatorname{Hom}\left(\Gamma_{g}\right.$, Homeo $\left._{+}\left(S^{1}\right)\right)$ contained in $N_{i}$. The intersection $U$ of the sets $U_{i}$ is an open neighbor-

hood of $\rho$ contained in $\bigcap_{i=1}^{g} N_{i}$. We claim that there is an open neighborhood of $\rho$ contained in $N_{0}$ as well.

To see this, suppose for contradiction that we can find a sequence of representations $\eta_{n}$ in $\operatorname{Hom}\left(\Gamma_{g}\right.$, Homeo $\left._{+}\left(S^{1}\right)\right) \backslash N_{0}$ approaching $\rho$. Without loss of generality, we may assume all $\eta_{n}$ lie in $U$. Then each $c_{i}\left(\eta_{n}\right)$ has a periodic orbit $X_{i}\left(\eta_{n}\right)$ and after passing to a subsequence we may assume that each $X_{i}\left(\eta_{n}\right)$ converges to a $k$-tuple $Y_{i}$, which will be a periodic orbit for $c_{i}(\rho)$. Now Corollary 4.8 implies that the periodic orbits $Y_{i}$ have the same combinatorial structure as the $X_{i}(\rho)$ in the definition of "good", and hence so do the sets $X_{i}\left(\eta_{n}\right)$ for $n$ sufficiently large. Thus, for large $n$, the representation $\eta_{n}$ is good, contradicting our assumption.

\subsection{Rotation rigidity for generators}

We return to work with the maximal PSL ${ }^{(\mathrm{k})}$ representation $\rho_{0}$ from Section 5.1, and will show that the rotation number of a single element of our standard generating set is constant on the connected component of $\rho_{0}$.

Proposition 5.11 (Rotation rigidity for a single generator). Let $N$ be the set defined in Section 5.2, and let $N^{\prime}$ be the connected component of $N$ containing $\rho_{0}$. Then $N^{\prime}$ is a connected component of $\operatorname{Hom}\left(\Gamma_{g}, \mathrm{Homeo}_{+}\left(S^{1}\right)\right)$ and $\operatorname{rot}\left(\rho\left(a_{g}\right)\right)=0$ for all $\rho \in N^{\prime}$.

Proof. By definition, $N$ is an intersection of closed sets, so $N^{\prime}$ is closed. To show that $N^{\prime}$ is also open, consider any $\rho \in N^{\prime}$. Since $\rho$ lies in the same component of $\operatorname{Hom}\left(\Gamma_{g}, \operatorname{Homeo}_{+}\left(S^{1}\right)\right)$ as $\rho_{0}$, we have $\mathrm{e}(\rho)=\mathrm{e}\left(\rho_{0}\right)=\frac{2 g-2}{k}$. It follows from Lemma 3.1 and the definition of Euler number that

$$
\tilde{\operatorname{rot}}\left(\left[\rho\left(a_{1}\right), \rho\left(b_{1}\right)\right] \ldots\left[\rho\left(a_{g-1}\right), \rho\left(b_{g-1}\right)\right]\right)+\tilde{\operatorname{rot}}\left[\rho\left(a_{g}\right), \rho\left(b_{g}\right)\right]=\frac{2 g-2}{k}
$$

From this and the inequalities $R_{i}(\rho) \leq 1 / k$ for $\rho \in N_{i}$, and $R(\rho) \leq \frac{2 g-3}{k}$ for $\rho \in N_{0}$, we can conclude that rot $\left[\rho\left(a_{g}\right), \rho\left(b_{g}\right)\right]=1 / k$ and rot $\left(\left[\rho\left(a_{1}\right), \rho\left(b_{1}\right)\right] \ldots\left[\rho\left(a_{g-1}\right), \rho\left(b_{g-1}\right)\right]\right)=\frac{2 g-3}{k}$. Thus, by Propositions 5.3 and 5.10, there is an open neighborhood of $\rho$ contained in $N$ and hence also in $N^{\prime}$. This proves that $N^{\prime}$ is a connected component of $\operatorname{Hom}\left(\Gamma_{g}, \operatorname{Homeo}_{+}\left(S^{1}\right)\right)$. Moreover, we have also just shown that $\operatorname{rot}\left[\rho\left(a_{g}\right), \rho\left(b_{g}\right)\right]=1 / k$ holds for all $\rho \in N^{\prime}$. Assume now for contradiction that $\operatorname{rot}\left(\rho\left(a_{g}\right)\right) \neq \operatorname{rot}\left(\rho_{0}\left(a_{g}\right)\right)$ for some $\rho \in N^{\prime}$. By continuity of rot, there exists a representation $\rho^{\prime} \in N^{\prime}$ such that $\operatorname{rot}\left(\rho^{\prime}\left(a_{g}\right)\right)$ is either irrational or is rational of the form $p / q$ with $q>k$. In either case, Lemma 2.4 implies that $\tilde{r} o t\left[\rho^{\prime}\left(a_{g}\right), \rho^{\prime}\left(b_{g}\right)\right]<1 / k$, a contradiction.

\subsection{Reduction to the case $\operatorname{rot}\left(\rho_{0}\left(a_{i}\right)\right)=0$ : a Euclidean algorithm trick}

Assuming now that $\operatorname{rot}\left(\rho_{0}\left(a_{i}\right)\right)=\frac{m_{i}}{k}$ for some $m \neq 0$, we show how to define a continuous map $\Phi: \operatorname{Hom}\left(\Gamma_{g}, \operatorname{Homeo}_{+}\left(S^{1}\right)\right) \rightarrow \operatorname{Hom}\left(\Gamma_{g}, \operatorname{Homeo}_{+}\left(S^{1}\right)\right)$ such that $\Phi\left(\rho_{0}\left(a_{i}\right)\right)$ is a maximal PSL ${ }^{(\mathrm{k})}$ representation with $\operatorname{rot}\left(\Phi\left(\rho_{0}\left(a_{i}\right)\right)\right)=0$. Since $\operatorname{rot}\left(\Phi\left(\rho_{0}\left(a_{i}\right)\right)\right)=0$, the results of Section 5.3 apply 


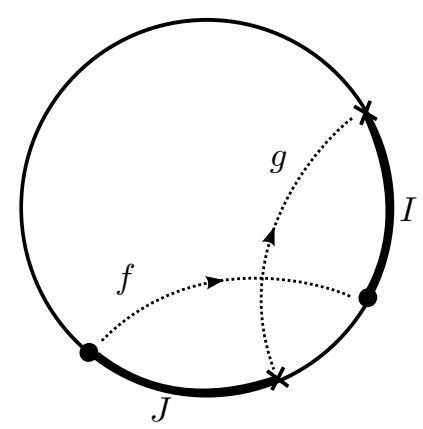

Figure 7: A pair of crossed homeomorphisms

to $\Phi\left(\rho_{0}\right)$, showing that the rotation number of the image of $a_{i}$ is constant on the connected component of $\Phi\left(\rho_{0}\right)$. Additional properties of $\Phi$ will let us conclude that the rotation number of the image of $a_{i}$ is constant on the component containing $\rho_{0}$ as well.

We start with a characterization of pairs $(f, g) \in \mathrm{PSL}^{(\mathrm{k})} \times \mathrm{PSL}^{(\mathrm{k})}$ such that $f=\rho\left(a_{i}\right)$ and $g=\rho\left(b_{i}\right)$ for a pair $\left(a_{i}, b_{i}\right)$ in a standard generating set and a maximal $\mathrm{PSL}^{(\mathrm{k})}$ representation $\rho$.

Definition 5.12 (crossed pair). Call a pair $(f, g) \in \mathrm{PSL}^{(\mathrm{k})} \times \mathrm{PSL}^{(\mathrm{k})}$ crossed if the projections $\bar{f}$ and $\bar{g}$ of $f$ and $g$ to $\operatorname{PSL}(2, \mathbb{R})$ are hyperbolic elements with intersecting (i.e. crossed) axes, with intersection number -1 .

In particular, for any maximal PSL ${ }^{(\mathrm{k})}$ representation $\rho$, the images of a pair of standard generators $\left(\rho\left(a_{i}\right), \rho\left(b_{i}\right)\right)$ form a crossed pair.

One crossed pair can be used to build another:

Lemma 5.13. Let $(f, g)$ be a crossed pair. Then $(f g, g)$ and $(f, g f)$ are crossed pairs also.

Proof. Let $f$ and $g$ be crossed, with projections $\bar{f}$ and $\bar{g} \in \operatorname{PSL}(2, \mathbb{R})$. The dynamics of the action of $\bar{f}$ and $\bar{g}$ on $S^{1}$ is as in Figure 7 below. Let $I \subset S^{1}$ be the closed interval bounded by the attracting fixed points of $\bar{f}$ and $\bar{g}$, and $J$ the closed interval bounded by the repelling fixed points, as in the figure. Then $\bar{f} \bar{g}(I) \subset I$ and $(\bar{f} \bar{g})^{-1}(J) \subset J$. It follows that $\bar{f} \bar{g}$ has an attracting fixed point in $I$ and a repelling fixed point in $J$, so its axis crosses the axis of $\bar{g}$. The same argument shows that the axis of $\bar{g} \bar{f}$ crosses the axis of $\bar{g}$.

The next lemma is an elementary result on rotation numbers of positive words. Recall that we use the terminology word in $a$ and $b$ to mean a word $w(a, b)$ in the letters $a$ and $b$, (and not $a^{-1}$ or $\left.b^{-1}\right)$, in other words, $w(a, b)$ is an element of the positive semigroup generated by $a$ and $b$.

Lemma 5.14. Let $(f, g)$ be a crossed pair, with $\operatorname{rot}(f)=\operatorname{rot}(g)=0$. Then any word $w=w(f, g)$ has $\operatorname{rot}(w)=0$.

Proof. Let $(f, g)$ be a crossed pair with projections $\bar{f}$ and $\bar{g} \in \operatorname{PSL}(2, \mathbb{R})$. Let $I \subset S^{1}$ be an interval as in Figure 7, so $\bar{f}(I) \subset I$ and $\bar{g}(I) \subset I$. Let $I^{\prime}$ be a single connected interval in the $k$-fold cover of $S^{1}$ that is a lift of $I$. Since $\operatorname{rot}(f)=\operatorname{rot}(g)=0$, it follows that $f\left(I^{\prime}\right) \subset I^{\prime}$ and $g\left(I^{\prime}\right) \subset I^{\prime}$, Thus, $w(f, g)\left(I^{\prime}\right) \subset I^{\prime}$ and so $w$ has a fixed point in $I^{\prime}$.

Now we can prove the main result of this section.

Proposition 5.15 (Euclidean algorithm for rotation numbers). Let $(a, b) \in \mathrm{PSL}^{(\mathrm{k})} \times \mathrm{PSL}^{(\mathrm{k})}$ be a crossed pair. There exist words $u=u(a, b)$ and $v=v(a, b)$ such that

i) $[u, v]=[a, b]$

ii) $\operatorname{rot}(u)=0$ 
iii) $u$ and $v$ are a crossed pair.

Proof. We will make repeated use of the following elementary algebraic computation, which applies to any commutator.

$$
[f, g]=\left[f, g f^{n}\right]=\left[f g^{n}, g\right] \text { for any } n \in \mathbb{Z}
$$

Let $m=k \operatorname{rot}(a)$ and $n=k \operatorname{rot}(b)$. Let $r: S^{1} \rightarrow S^{1}$ denote the order $k$ rigid rotation with rotation number $\frac{1}{k}$, which commutes with $a$ and $b$. Let $a^{\prime}=a r^{k-m}$ and $b^{\prime}=b r^{k-n}$. Note that $\operatorname{rot}\left(a^{\prime}\right)=\operatorname{rot}\left(b^{\prime}\right)=0$, and that $\left(a^{\prime}, b^{\prime}\right)$ is a crossed pair in $\mathrm{PSL}^{(\mathrm{k})}$.

As a warm up for the rest of the proof, we do a simple computation. Let $\alpha_{1}, \beta_{1}, \ldots \alpha_{s}, \beta_{s}$ be integers. Then

$$
a^{\alpha_{1}} b^{\beta_{1}} \ldots a^{\alpha_{s}} b^{\beta_{s}}=\left(a^{\prime}\right)^{\alpha_{1}}\left(b^{\prime}\right)^{\beta_{1}} \ldots\left(a^{\prime}\right)^{\alpha_{s}}\left(b^{\prime}\right)^{\beta_{s}} r^{\left(m\left(\alpha_{1}+\ldots+\alpha_{s}\right)+n\left(\beta_{1}+\ldots+\beta_{s}\right)\right)}
$$

By Lemma 5.14, $\operatorname{rot}\left(\left(a^{\prime}\right)^{\alpha_{1}}\left(b^{\prime}\right)^{\beta_{1}} \ldots\left(a^{\prime}\right)^{\alpha_{s}}\left(b^{\prime}\right)^{\beta_{s}}\right)=0$, so

$$
\operatorname{rot}\left(a^{\alpha_{1}} b^{\beta_{1}} \ldots a^{\alpha_{s}} b^{\beta_{s}}\right)=\frac{m\left(\alpha_{1}+\ldots+\alpha_{s}\right)+n\left(\beta_{1}+\ldots+\beta_{s}\right)}{k} .
$$

This kind of computation lets us reduce the rotation number of $a$ while preserving the commutator $[a, b]$, as follows. First, use the (standard) Euclidean algorithm to produce a sequence of pairs of integers

$$
(m, n)=\left(m_{0}, n_{0}\right),\left(m_{0}, n_{1}\right),\left(m_{1}, n_{1}\right),\left(m_{1}, n_{2}\right), \ldots
$$

with $n_{i}=n_{i-1}-q_{i} m_{i-1}$ and $m_{i}=m_{i-1}-p_{i} n_{i}$, terminating after $d$ steps with a pair either of the form $\left(m_{d}, 0\right)$ or $\left(0, n_{d}\right)$. Choose $\bar{q}_{i}>0$ and $\bar{p}_{i}>0$ such that $\bar{q}_{i} \equiv-q_{i} \bmod k$, and $\bar{p}_{i} \equiv-p_{i}$ $\bmod k$, and consider the sequence of pairs of elements of PSL ${ }^{(\mathrm{k})}$

$$
\left(f_{0}, g_{0}\right),\left(f_{0}, g_{1}\right),\left(f_{1}, g_{1}\right),\left(f_{1}, g_{2}\right) \ldots
$$

defined recursively by setting $\left(f_{0}, g_{0}\right)=(a, b)$ and

$$
\begin{aligned}
g_{i} & =g_{i-1} f_{i-1}^{\bar{q}_{i}} \\
f_{i} & =f_{i-1} g_{i}^{\bar{p}_{i}}
\end{aligned}
$$

The sequence terminates at step $d$ with a pair of words $(u, v)=(u(a, b), v(a, b))$. The reader may find it instructive to look at the first few terms of the sequence:

$$
(a, b),\left(a, b a^{\bar{q}_{1}}\right),\left(a\left(b a^{\bar{q}_{1}}\right)^{\bar{p}_{1}}, b a^{\bar{q}_{1}}\right), \ldots
$$

The recursive definition of $f_{i}$ and $g_{i}$ together with Equation (10) implies that for each $i$ we have

$$
\left[f_{i}, g_{i}\right]=\left[f_{i}, g_{i+1}\right]=\left[f_{i+1}, g_{i+1}\right] .
$$

It follows that $[u, v]=[a, b]$. Moreover, Lemma 5.13 implies inductively that each pair in the sequence is a crossed pair. Finally, the calculation in our warm-up (equation (11)) shows that $\operatorname{rot}\left(f_{i}\right)=m_{i} / k$ and $\operatorname{rot}\left(g_{i}\right)=n_{i} / k$ (recall that rotation numbers take values in $\mathbb{R} / \mathbb{Z}$ ). Thus, the final pair $(u, v)$ satisfies either $\operatorname{rot}(u)=0 \operatorname{or} \operatorname{rot}(v)=0$. If $\operatorname{rot}(u)=0$, we are done. If instead $\operatorname{rot}(v)=0$, replace $(u, v)$ with the pair $\left(u v u^{k-1}, v u^{k-1}\right)$. Lemma 5.13 implies first that $\left(u, v u^{k-1}\right)$ is a crossed pair, and then so is $\left(u v u^{k-1}, v u^{k-1}\right)$. The commutators satisfy $\left[u v u^{k-1}, v u^{k-1}\right]=[u, v]=[a, b]$ and now we have $\operatorname{rot}\left(u v u^{k-1}\right)=\operatorname{rot}(v)=0$, as desired. 
We can now carry out the reduction to the case $\operatorname{rot}\left(\rho_{0}\left(a_{i}\right)\right)=0$, justifying this assumption in Sections 5.1 through 5.3. Let $\rho_{0}$ be any maximal PSL ${ }^{(\mathrm{k})}$ representation. Then each pair $\left(\rho_{0}\left(a_{i}\right), \rho_{0}\left(b_{i}\right)\right)$ is a crossed pair. Use Proposition 5.15 to produce words $u_{i}(a, b)$ and $v_{i}(a, b)$ in the letters $a$ and $b$ such that - letting $u_{i}(\rho)$ denote $u_{i}\left(\rho\left(a_{i}\right), \rho\left(b_{i}\right)\right)$ and $v_{i}(\rho)$ denote $v_{i}\left(\rho\left(a_{i}\right), \rho\left(b_{i}\right)\right)$ we have

i) $\left[u_{i}(\rho), v_{i}(\rho)\right]=\left[\rho\left(a_{i}\right), \rho\left(b_{i}\right)\right]$ for any $\rho$, and

ii) $\operatorname{rot}\left(u_{i}\left(\rho_{0}\right)\right)=0$.

Define a function $\Phi: \operatorname{Hom}\left(\Gamma_{g}, \operatorname{Homeo}_{+}\left(S^{1}\right)\right) \rightarrow \operatorname{Hom}\left(\Gamma_{g}, \operatorname{Homeo}_{+}\left(S^{1}\right)\right)$ by $\Phi(\rho)\left(a_{i}\right)=u_{i}(\rho)$ and $\Phi(\rho)\left(b_{i}\right)=v_{i}(\rho)$, for each $a_{i}$ and $b_{i}$ in a standard generating set. This function is continuous, and the property i) above implies that it is well defined on $\Gamma_{g}$. Let $\eta_{0}=\Phi\left(\rho_{0}\right)$. Then the image of $\eta_{0}$ lies in $\mathrm{PSL}^{(\mathrm{k})}$. Moreover, one can deduce from property i) and the fact that $\tilde{\operatorname{rot}}\left[\rho_{0}\left(a_{i}\right), \rho_{0}\left(b_{i}\right)\right]=1 / k$ that $\left[\tilde{u}_{i}(\rho), \tilde{v}_{i}(\rho)\right]=\left[\tilde{\rho}_{0}\left(a_{i}\right), \tilde{\rho}_{0}\left(b_{i}\right)\right]$, so $\mathrm{e}\left(\eta_{0}\right)=\mathrm{e}\left(\rho_{0}\right)=\frac{2 g-2}{k}$. Since $\operatorname{rot}\left(\eta_{0}\left(a_{i}\right)\right)=\operatorname{rot}\left(u_{i}\left(\rho_{0}\right)\right)=0$ for all $i$, the results of Sections 5.1 through 5.3 above apply here to show that $\operatorname{rot}\left[\eta\left(a_{g}\right), \eta\left(b_{g}\right)\right]=1 / k$ for all $\eta$ in the connected component of $\eta_{0}$.

Suppose that $\rho$ is a representation in the same component as $\rho_{0}$. Since $\Phi$ is continuous, $\Phi(\rho)$ lies in the same component as $\eta_{0}$, so

$$
\tilde{\operatorname{rot}}\left[\rho\left(a_{g}\right), \rho\left(b_{g}\right)\right]=\tilde{\operatorname{rot}}\left[\Phi(\rho)\left(a_{g}\right), \Phi(\rho)\left(b_{g}\right)\right]=\tilde{\operatorname{rot}}\left[\eta\left(a_{g}\right), \eta\left(b_{g}\right)\right]=1 / k .
$$

Since rot $\left[\rho\left(a_{g}\right), \rho\left(b_{g}\right)\right]=1 / k$ for all $\rho$ in the connected component of $\rho_{0}$, the argument from the end of Proposition 5.11 now applies to show that $\operatorname{rot}\left(\rho\left(a_{g}\right)\right)=\operatorname{rot}\left(\rho_{0}\left(a_{g}\right)\right)$ for all $\rho$ in the connected component of $\rho_{0}$ in $\operatorname{Hom}\left(\Gamma_{g}, \operatorname{Homeo}_{+}\left(S^{1}\right)\right)$.

In summary, we have just shown the following.

Proposition 5.16 (Rotation rigidity for generators, general version). Let $\rho_{0}$ be a maximal PSL ${ }^{(\mathrm{k})}$ representation, and $\left\{a_{1}, b_{1}, \ldots a_{g}, b_{g}\right\}$ a standard generating set for $\Gamma_{g}$. Let $X \subset \operatorname{Hom}\left(\Gamma_{g}\right.$, Homeo $\left._{+}\left(S^{1}\right)\right)$ be a connected component containing a maximal PSL ${ }^{(\mathrm{k})}$ representation $\rho_{0}$. Then $\operatorname{rot}\left(\rho\left(a_{g}\right)\right)=\operatorname{rot}\left(\rho_{0}\left(a_{g}\right)\right)$ for all $\rho \in X$.

\subsection{Finishing the proof of Theorem 1.4}

Theorem 1.4 will follow from Proposition 5.16 using a covering trick due to Matsumoto in [9]. Let $X$ be a component of $\operatorname{Hom}\left(\Gamma_{g}\right.$, Homeo+ $\left.\left(S^{1}\right)\right)$ that contains a maximal PSL ${ }^{(\mathrm{k})}$ representation $\rho_{0}$, and let $\rho \in X$. Let $\gamma \in \Gamma_{g}$, and let $\alpha$ be a curve on $\Sigma_{g}$ representing $\gamma$. If $\alpha$ is a nonseparating simple closed curve, then we may include $\gamma$ into a standard generating set for $\Gamma_{g}$, in which case Proposition 5.16 implies that $\operatorname{rot}(\rho(\gamma))=\operatorname{rot}\left(\rho_{0}(\gamma)\right)$.

If $\alpha$ is not a simple closed curve, we may take a finite cover $\Sigma_{g^{\prime}}$ of $\Sigma_{g}$ such that $\alpha$ lifts to a nonseparating simple closed curve $\beta$ (such a cover always exists - Scott's theorem in [11] implies that $\alpha$ can be lifted to a simple closed curve in some finite cover, and taking a further cover we can ensure that the lift is nonseparating). Let $a_{g^{\prime}} \in \pi_{1}\left(\Sigma_{g^{\prime}}\right)$ represent this lift, and include $a_{g^{\prime}}$ in a standard generating set $\left\{a_{1}, b_{1} \ldots a_{g^{\prime}}, b_{g^{\prime}}\right\}$ for $\Sigma^{\prime}$. If the degree of the cover $\Sigma_{g^{\prime}} \stackrel{\pi}{\rightarrow} \Sigma_{g}$ is $m$, then $g^{\prime}=m(g-1)+1$.

Consider now the (continuous) function $\pi^{*}: \operatorname{Hom}\left(\Gamma_{g}, \operatorname{Homeo}_{+}\left(S^{1}\right)\right) \rightarrow \operatorname{Hom}\left(\Gamma_{g^{\prime}}, \operatorname{Homeo}_{+}\left(S^{1}\right)\right)$ defined by $\rho \mapsto \rho \circ \pi_{*}$. The Euler number is multiplicative with respect to covers, so we have

$$
\mathrm{e}\left(\pi^{*}(\rho)\right)=m \mathrm{e}(\rho)=\frac{m(2 g-2)}{k}=\frac{2 g^{\prime}-2}{k}
$$

In particular, $\pi^{*}\left(\rho_{0}\right)$ is also a maximal PSL ${ }^{(\mathrm{k})}$ representation. Since $\pi^{*}(\rho)$ lies in the same component of $\operatorname{Hom}\left(\Gamma_{g^{\prime}}\right.$, Homeo $\left._{+}\left(S^{1}\right)\right)$ as $\pi^{*}\left(\rho_{0}\right)$, Proposition 5.16 implies that

$$
\operatorname{rot}\left(\pi^{*}\left(\rho_{0}\left(a_{g^{\prime}}\right)\right)\right)=\operatorname{rot}\left(\pi^{*}\left(\rho\left(a_{g^{\prime}}\right)\right)\right) .
$$


Since $\pi_{*}\left(a_{g^{\prime}}\right)=\alpha$, we conclude that $\operatorname{rot}\left(\rho_{0}(\alpha)\right)=\operatorname{rot}(\rho(\alpha))$ as desired. This concludes the proof of Theorem 1.4.

\section{Proof of Theorem 1.3}

Theorem 1.3 will now follow from Theorem 1.4. We recall the statement here.

Theorem 1.3. For each nontrivial divisor $k$ of $2 g-2$, there are at least $k^{2 g}+1$ components of $\operatorname{Hom}\left(\Gamma_{g}, \mathrm{Homeo}_{+}\left(S^{1}\right)\right)$ consisting of representations with Euler number $\frac{2 g-2}{k}$.

In particular, two representations into $\mathrm{PSL}^{(\mathrm{k})}$ that lie in different components of $\operatorname{Hom}\left(\Gamma_{g}, \mathrm{PSL}^{(\mathrm{k})}\right)$ necessarily lie in different components of $\operatorname{Hom}\left(\Gamma_{g}, \operatorname{Homeo}_{+}\left(S^{1}\right)\right)$.

Proof. Let $k$ be a nontrivial divisor of $2 g-2$. As explained in the proof of Proposition 2.10, representations $\rho: \Gamma_{g} \rightarrow \mathrm{PSL}^{(\mathrm{k})}$ with Euler number $\mathrm{e}(\rho)=\frac{2 g-2}{k}$ are precisely the lifts of faithful Fuchsian representations $\nu: \Gamma_{g} \rightarrow \operatorname{PSL}(2, \mathbb{R})$. Each representation $\nu$ has $k^{2 g}$ lifts; these can be distinguished by reading the rotation numbers of each of a standard set of generators, which may take any value in $\left\{0, \frac{1}{k}, \ldots, \frac{k-1}{k}\right\}$.

Goldman's theorem (Theorem 1.5) states that the rotation numbers of the generators are a complete invariant of connected components of $\operatorname{Hom}\left(\Gamma_{g}, \mathrm{PSL}^{(\mathrm{k})}\right)$ - there are $k^{2 g}$ components of $\operatorname{Hom}\left(\Gamma_{g}, \mathrm{PSL}^{(\mathrm{k})}\right)$, distinguished by the rotation numbers of a standard set of generators. Thus, if $\rho_{1}$ and $\rho_{2}$ are representations that lie in different components of $\operatorname{Hom}\left(\Gamma_{g}, \mathrm{PSL}^{(\mathrm{k})}\right)$, then the rotation number of some generator differs under $\rho_{1}$ and $\rho_{2}$, and so by Theorem 1.4, $\rho_{1}$ and $\rho_{2}$ must lie in different components of $\operatorname{Hom}\left(\Gamma_{g}, \mathrm{Homeo}_{+}\left(S^{1}\right)\right)$.

Finally, to conclude that $\operatorname{Hom}\left(\Gamma_{g}, \mathrm{HomeO}_{+}\left(S^{1}\right)\right)$ has at least $k^{2 g}+1$ connected components consisting of representations with Euler number $\frac{2 g-2}{k}$, it suffices to exhibit a representation $\rho: \Gamma_{g} \rightarrow$ Homeo $_{+}\left(S^{1}\right)$ with e $(\rho)=\frac{2 g-2}{k}$ and $\rho\left(a_{g}\right) \neq \frac{m}{k}$ for any integer $m$. Such a representation can be constructed as follows. Take a representation $\mu: \Gamma_{g-1} \rightarrow \operatorname{Homeo}_{+}\left(S^{1}\right)$ with $\mathrm{e}(\mu)=\frac{2 g-2}{k}$ and extend this to a representation $\rho: \Gamma_{g} \rightarrow \operatorname{Homeo}_{+}\left(S^{1}\right)$ as follows: If $\left\{a_{1}, b_{1}, \ldots a_{g-1}, b_{g-1}\right\}$ is a standard generating set for $\Gamma_{g-1}$, then we can take $\left\{a_{1}, b_{1}, \ldots a_{g}, b_{g}\right\}$ a standard generating set for $\Gamma_{g}$, and define $\rho$ on generators by

$$
\begin{array}{ll}
\rho\left(a_{i}\right)=\nu\left(a_{i}\right), & \rho\left(b_{i}\right)=\nu\left(b_{i}\right), \quad \text { for } i=1,2, \ldots, g-1 \\
\rho\left(a_{g}\right)=r_{\alpha}, & \rho\left(b_{g}\right)=r_{\beta}
\end{array}
$$

where $r_{\alpha}$ and $r_{\beta}$ are rigid rotations by arbitrary angles $\alpha$ and $\beta$, with $2 \pi k \beta \notin \mathbb{Z}$. Since $\left[r_{\alpha}, r_{\beta}\right]=\mathrm{id}$, we have

$$
\left[\tilde{\rho}\left(a_{1}\right), \tilde{\rho}\left(b_{1}\right)\right] \ldots\left[\tilde{\rho}\left(a_{g-1}\right), \tilde{\rho}\left(b_{g-1}\right)\right]\left[\tilde{r}_{\alpha}, \tilde{r}_{\beta}\right]=\left[\tilde{\rho}\left(a_{1}\right), \tilde{\rho}\left(b_{1}\right)\right] \ldots\left[\tilde{\rho}\left(a_{g-1}\right), \tilde{\rho}\left(b_{g-1}\right)\right],
$$

a translation by $\frac{2 g-2}{k}$. Thus, $\rho$ is a representation and has Euler number e $(\rho)=\frac{2 g-2}{k}$.

\section{Semi-conjugate representations: Theorem 1.2}

In this section we recall the notion of semi-conjugacy and prove Theorem 1.2. The reader should note that use of the term "semi-conjugacy" for representations to $\operatorname{Homeo}_{+}\left(S^{1}\right)$ in the existing literature is inconsistent. We will use the following definition, which appears in [4].

Definition 7.1 (Degree 1 monotone map). A map $h: S^{1} \rightarrow S^{1}$ is called a degree 1 monotone map if it is continuous and admits a lift $\tilde{h}: \mathbb{R} \rightarrow \mathbb{R}$, equivariant with respect to integer translations, and nondecreasing on $\mathbb{R}$. 
Definition 7.2 (Semi-conjugacy of representations). Let $\Gamma$ be any group. Two representations $\rho_{1}$ and $\rho_{2}$ in $\operatorname{Hom}\left(\Gamma, \mathrm{Homeo}_{+}\left(S^{1}\right)\right)$ are semi-conjugate if there is a degree one monotone map $h: S^{1} \rightarrow S^{1}$ such that $\rho_{1}(\gamma) \circ h=h \circ \rho_{2}(\gamma)$ for all $\gamma \in \Gamma$.

Semi-conjugacy is not an equivalence relation because it is not symmetric. However, there is a relatively simple description of representations that lie in the same class under the equivalence relation generated by semi-conjugacy. This description is due to Calegari and Dunfield.

Proposition 7.3 (Definition 6.5 and Lemma 6.6 of [2]). Two representations $\rho_{1}$ and $\rho_{2}$ in $\operatorname{Hom}\left(\Gamma\right.$, Homeo $\left._{+}\left(S^{1}\right)\right)$ lie in the same semi-conjugacy class if and only if there is a third representation $\rho: \Gamma \rightarrow$ Homeo $_{+}\left(S^{1}\right)$ and degree one monotone maps $h_{1}$ and $h_{2}$ of $S^{1}$ such that $\rho_{i} \circ h_{i}=h_{i} \circ \rho$.

In [5], Ghys showed that two representations in $\operatorname{Hom}\left(\Gamma, \operatorname{Homeo}_{+}\left(S^{1}\right)\right)$ lie in the same semiconjugacy class if and only if they have the same bounded integer Euler class in $H_{b}^{2}(\Gamma ; \mathbb{Z})$. Matsumoto later translated this into a condition defined purely in terms of (lifted) rotation numbers. Before we state this condition, note that for any two elements $a, b \in \operatorname{Homeo}_{+}\left(S^{1}\right)$ with lifts $\tilde{a}$ and $\tilde{b}$ in $\mathrm{Homeo}_{\mathbb{Z}}(\mathbb{R})$, the number

$$
\tau(a, b):=\tilde{\operatorname{rot}}(\tilde{a} \tilde{b})-\tilde{\operatorname{rot}}(\tilde{a})-\tilde{\operatorname{rot}}(\tilde{b})
$$

does not depend on the choice of lifts $\tilde{a}$ and $\tilde{b}$.

Proposition 7.4 (Matsumoto, [8]). Let $\Gamma$ be a group with generating set $\left\{\gamma_{i}\right\}$. Two representations $\rho_{1}$ and $\rho_{2}$ in $\operatorname{Hom}\left(\Gamma, \operatorname{Homeo}_{+}\left(S^{1}\right)\right)$ lie in the same semi-conjugacy class if and only if the following two conditions hold

i) Each generator $\gamma_{i}$ of $\Gamma$ satisfies $\operatorname{rot}\left(\rho_{1}(\gamma)\right)=\operatorname{rot}\left(\rho_{2}(\gamma)\right)$.

ii) Each pair of elements $\gamma$ and $\gamma^{\prime}$ in $\Gamma$ satisfies $\tau\left(\rho_{1}(\gamma), \rho_{1}\left(\gamma^{\prime}\right)\right)=\tau\left(\rho_{2}(\gamma), \rho_{2}\left(\gamma^{\prime}\right)\right)$.

As a corollary of Matsumoto's condition we have the following. Recall that for $\gamma \in \Gamma$, we let $\operatorname{rot}_{\gamma}: \operatorname{Hom}\left(\Gamma\right.$, Homeo $\left._{+}\left(S^{1}\right)\right) \rightarrow \mathbb{R} / \mathbb{Z}$ be defined by $\operatorname{rot}_{\gamma}(\rho)=\operatorname{rot}(\rho(\gamma))$.

Corollary 7.5. Let $\Gamma$ be any group and $U \subset \operatorname{Hom}\left(\Gamma, \mathrm{Homeo}_{+}\left(S^{1}\right)\right)$ a connected set. If for all $\gamma \in \Gamma$ the function $\operatorname{rot}_{\gamma}$ is constant on $U$, then $U$ consists of a singe semi-conjugacy class.

Proof. By assumption, condition i) of Proposition 7.4 is automatically satisfied for any two representations in $U$. To see that ii) is satisfied, fix any $\gamma$ and $\gamma^{\prime}$ in $\Gamma$, and define a function $F_{\gamma, \gamma^{\prime}}: U \times U \rightarrow \mathbb{R}$ by

$$
F_{\gamma, \gamma^{\prime}}(\rho, \nu)=\tau\left(\rho(\gamma) \rho\left(\gamma^{\prime}\right)\right)-\tau\left(\nu(\gamma), \nu\left(\gamma^{\prime}\right)\right) .
$$

Then $F_{\gamma, \gamma^{\prime}}$ is clearly continuous. We will show it is integer valued, hence constant. Since $F_{\gamma, \gamma^{\prime}}(\rho, \rho)=0$, it will follow that condition ii) is satisfied.

That $F_{\gamma, \gamma^{\prime}}$ is constant comes from the fact that $\operatorname{rot}_{\gamma}$ and $\operatorname{rot}_{\gamma^{\prime}}$ are constant on $U$. By definition,

$$
F_{\gamma, \gamma^{\prime}}(\rho, \nu)=\tilde{\operatorname{rot}}\left(\tilde{\rho}(\gamma) \tilde{\rho}\left(\gamma^{\prime}\right)\right)-\tilde{\operatorname{rot}}\left(\tilde{\nu}(\gamma) \tilde{\nu}\left(\gamma^{\prime}\right)\right)-\tilde{\operatorname{rot}}(\tilde{\rho}(\gamma))+\tilde{\operatorname{rot}}(\tilde{\rho}(\nu))-\tilde{\operatorname{rot}}\left(\tilde{\rho}\left(\gamma^{\prime}\right)\right)+\tilde{\operatorname{rot}}\left(\tilde{\nu}\left(\gamma^{\prime}\right)\right)
$$

Taken $\bmod \mathbb{Z}$, this expression becomes

$$
\operatorname{rot}\left(\rho(\gamma) \rho\left(\gamma^{\prime}\right)\right)-\operatorname{rot}\left(\nu(\gamma) \nu\left(\gamma^{\prime}\right)\right)-\operatorname{rot}(\rho(\gamma))+\operatorname{rot}(\rho(\nu))-\operatorname{rot}\left(\rho\left(\gamma^{\prime}\right)\right)+\operatorname{rot}\left(\nu\left(\gamma^{\prime}\right)\right),
$$

which is zero, since $\operatorname{rot}_{\gamma}$ and $\operatorname{rot}_{\gamma^{\prime}}$ are constant on $U$. 
Combining Theorem 1.4 with Corollary 7.5, we conclude that if $X$ is a connected component of $\operatorname{Hom}\left(\Gamma_{g}, \mathrm{Homeo}_{+}\left(S^{1}\right)\right)$ that contains a maximal PSL ${ }^{(\mathrm{k})}$ representation (equivalently, a geometric representation by Proposition 2.11), then all representations in $X$ lie in the same semi-conjugacy class. Thus, to finish the proof of Theorem 1.2, it suffices to prove that representations in the same semi-conjugacy class always lie in the same component of $\operatorname{Hom}\left(\Gamma_{g}, \operatorname{Homeo}_{+}\left(S^{1}\right)\right)$. In fact, they always lie in the same path-component.

Proposition 7.6. If $\rho_{1}$ and $\rho_{2}$ lie in the same semi-conjugacy class, then there is a continuous path $\rho_{t}$ in $\operatorname{Hom}\left(\Gamma_{g}, \mathrm{Homeo}_{+}\left(S^{1}\right)\right)$ from $\rho_{0}$ to $\rho_{1}$.

Proof. Let $\rho: \Gamma_{g} \rightarrow$ Homeo $_{+}\left(S^{1}\right)$ and let $h_{i}: S^{1} \rightarrow S^{1}$ be monotone maps as in Proposition 7.3 with $h_{i} \circ \rho=\rho_{i} \circ h_{i}$. We will construct a path from $\rho_{1}$ to $\rho$ (and the same procedure will work to build a path from $\rho_{2}$ to $\rho$ ).

First, for $t \in[1 / 2,1)$ let $\hat{h}_{t}$ be a path of homeomorphisms of $S^{1}$ such that $\lim _{t \rightarrow 1} \hat{h}_{t}=h$. Then $\hat{h}_{1 / 2}$ is isotopic to the identity, so for $t \in[0,1 / 2]$ define $\hat{h}_{t}$ to be a path from id to $\hat{h}_{1 / 2}$. Now, for all $t \in[0,1)$ we may define $\rho_{t}$ by

$$
\rho_{t}(\gamma)=\hat{h}_{t} \rho(\gamma) \hat{h}_{t}^{-1}
$$

Note that $\rho_{0}=\rho$, and $\rho_{t}(\gamma) \rightarrow \rho_{1}(\gamma)$ as $t \rightarrow 1$ (using the fact that $h$ is surjective). Thus, $\rho_{t}$ gives a continuous path from $\rho_{1}$ to $\rho$, and the same kind of construction will clearly work for $\rho_{2}$.

This concludes our proof of Theorem 1.2.

\section{$8 \quad$ Rigidity and Flexibility}

Say that a representation $\rho \in \operatorname{Hom}\left(\Gamma_{g}, \operatorname{Homeo}_{+}\left(S^{1}\right)\right)$ is rigid if the connected component of $\operatorname{Hom}\left(\Gamma_{g}\right.$, Homeo $\left._{+}\left(S^{1}\right)\right)$ containing $\rho$ consists of a single semi-conjugacy class. (Proposition 7.4 and Corollary 7.5 imply that this is equivalent to the functions $\operatorname{rot}_{\gamma}$ being constant on the component containing $\rho$, for each $\gamma \in \Gamma_{g}$.) Theorem 1.2 states that geometric representations are rigid. We conjecture that these are effectively the only rigid representations in $\operatorname{Hom}\left(\Gamma_{g}, \operatorname{Homeo}_{+}\left(S^{1}\right)\right)$.

Conjecture 8.1. Suppose that $\rho \in \operatorname{Hom}\left(\Gamma_{g}, \operatorname{Homeo}_{+}\left(S^{1}\right)\right)$ is rigid. Then $\mathrm{e}(\rho)=\frac{2 g-2}{k}$ for some $k$, and $\rho$ lies in the semi-conjugacy class of a representation with image in $\operatorname{PSL}^{(\mathrm{k})}$.

One can make an analogous statement for $\operatorname{Hom}\left(\Gamma_{g}, \operatorname{Diff}_{+}\left(S^{1}\right)\right)$ :

Conjecture 8.2. Suppose that $\rho \in \operatorname{Hom}\left(\Gamma_{g}, \operatorname{Diff}_{+}\left(S^{1}\right)\right)$ is rigid, meaning that the connected component of $\operatorname{Hom}\left(\Gamma_{g}, \operatorname{Diff}_{+}\left(S^{1}\right)\right)$ containing $\rho$ consists of a single semi-conjugacy class. Then $\mathrm{e}(\rho)=\frac{2 g-2}{k}$ for some $k$, and $\rho$ lies in the semi-conjugacy class of a representation with image in $\mathrm{PSL}^{(\mathrm{k})}$.

Progress on these conjectures, as well as related work on flexibility and rigidity of representations to $\operatorname{Hom}\left(\Gamma_{g}, \operatorname{Diff}_{+}\left(S^{1}\right)\right)$ is the subject of a forthcoming paper. For now, we note the following theorem of Goldman, which gives a quick answer to Conjecture 8.1 in the special case where $\rho$ has image in $\operatorname{PSL}^{(\mathrm{k})}$.

Proposition 8.3 (Goldman, see the proof of Lemma 10.5 in [6].). Let $\rho: \Gamma_{g} \rightarrow$ PSL $^{(\mathrm{k})}$ satisfy $|\mathrm{e}(\rho)|<\frac{2 g-2}{k}$. Then there is a representation $\nu$ in the same path component of $\operatorname{Hom}\left(\Gamma, \mathrm{PSL}^{(\mathrm{k})}\right)$ as $\rho$, and an element $\gamma \in \Gamma_{g}$ such that $\operatorname{rot}(\rho(\gamma)) \neq \operatorname{rot}(\nu(\gamma))$. 


\section{References}

[1] J. Bowden Contact structures, deformations and taut foliations. Preprint. arxiv:1304.3833v1

[2] D. Calegari, N. Dunfield Laminations and groups of homeomorphisms of the circle. Invent. Math. 152 no. 1 (2003) 149-204.

[3] D. Calegari, A. Walker. Ziggurats and rotation numbers. Journal of Modern Dynamics 5, no. 4 (2011) 711-746.

[4] E. Ghys Groups acting on the circle. L'Enseignement Mathématique, 47 (2001) 329-407.

[5] E. Ghys Groupes d'homèomorphismes du cercle et cohomologie bornèe. The Lefschetz centennial conference, Part III. Contemp. Math. 58 III, Amer. Math. Soc., Providence, RI, (1987) 81-106.

[6] W. Goldman Topological components of spaces of representations. Invent. Math. 93 no. 3 (1998) 557-607.

[7] J. Milnor On the existence of a connection with curvature zero. Comm. Math. Helv. 32 no. 1 (1958) 215-223.

[8] S. Matsumoto Numerical invariants for semi-conjugacy of homeomorphisms of the circle. Proc. AMS 96 no.1 (1986) 163-168.

[9] S. Matsumoto Some remarks on foliated $S^{1}$ bundles. Invent. math. 90 (1987) 343-358.

[10] A. Navas Groups of circle diffeomorphisms. Univ. Chicago press, 2011.

[11] P. Scott Subgroups of surface groups are almost geometric. J. London Math. Soc. (2) 17 no. 3 (1978) 555-565.

[12] J. Wood Bundles with totally disconnected structure group. Comm. Math. Helv. 51 (1971) 183-199.

Dept. of Mathematics

University of Chicago.

5734 University Ave.

Chicago, IL 60637.

E-mail: mann@math.uchicago.edu 